\title{
Distribution et habitats de Newbouldia laevis (P.Beauv.) Seemann ex Bureau et de Dracaena arborea (Willd.) Link dans les zones bioclimatiques du Bénin
}

\author{
Jhonn LOGBO $^{1,2^{*}}$, Paul YEDOMONHAN ${ }^{2}$, Brice TENTE $^{3}$ et Akpovi AKOEGNINOU ${ }^{2}$ \\ ${ }^{I}$ Ecole d'Horticulture et d'Aménagement des Espaces Verts (EHAEV), Université Nationale d'Agriculture \\ (UNA), BP 43, Kétou, Bénin. \\ ${ }^{2}$ Laboratoire de Botanique et d'Ecologie Végétale (LaBEV), Faculté des Sciences et Techniques (FAST), \\ Université d'Abomey-Calavi (UAC), 01 BP 4521 Cotonou, Bénin. \\ ${ }^{3}$ Département de Géographie et d'Aménagement du Territoire (DGAT), Université d'Abomey-Calavi (UAC), \\ 01 BP : 526 Cotonou; Bénin. \\ ${ }^{3}$ Laboratoire de Biogéographie et Expertise Environnementale (LABEE) ; Faculté des Sciences Humaines \\ (FASH), Université d'Abomey-Calavi (UAC), BP 677, Abomey-Calavi ; Bénin. \\ *Auteur Correspondant ; E-mail : jhonn_logbo@yahoo.fr; Tél. (+229) 96662190
}

\section{RÉSUMÉ}

Cette étude a permis de déterminer la distribution et les habitats potentiels de Newbouldia laevis et de Dracaena arborea dans les zones bioclimatiques du Bénin en vue de leur gestion durable. Trente relevés phytosociologiques ont été effectués dans les formations végétales. La matrice obtenue a permis de discriminer les forêts sacrées (74 genres ; 46 familles ; Richesse spécifique $=130 ; \mathrm{H}=5,92$ bits; $\mathrm{R}=0,97$ ) des champs/jachères (42 genres ; 28 familles ; Richesse spécifique $=69 ; \mathrm{H}=3,40$ bits; $\mathrm{R}=0,62$ ). Les densités moyennes de tiges sont passées en forêts sacrées de 64 à 31 et à 0 tiges.ha $^{-1}$; puis de 28 à 16 tiges.ha $^{-1}$ dans les champs/jachères chez $D$. arborea. Chez $N$. laevis, ces densités sont passées de 30 à 15 puis à 09 tiges.ha ${ }^{-1}$ en forêts sacrées et de 89 à 56 et à 13 tiges.ha ${ }^{-1}$ dans les champs/jachères respectivement en zones guinéo-congolaise, soudano-guinéenne et soudanienne. Les phanérophytes et les espèces guinéo-congolaises sont les plus dominants. D. arborea et $N$. laevis se conservent mieux respectivement en forêts sacrées et en champs/jachères. Leur introduction dans les programmes de reboisement et la protection de leurs habitats potentiels seraient un atout pour leur meilleure conservation et contribueront à formuler des recommandations soutenues pour leur valorisation au Bénin.

(C) 2020 International Formulae Group. All rights reserved.

Mots clés : Bénin, Distribution, Zones bioclimatiques, Newbouldia laevis, Dracaena arborea.

\section{Distribution and habitats of Newbouldia laevis (P.Beauv.) Seemann ex Bureau and Dracaena arborea (Willd.) Link in the bioclimatic zones of Benin}

\begin{abstract}
This study carried out in the three bioclimatic zones of Benin aims to determine the potential habitats and the geographic distributions of Newbouldia laevis and Dracaena arborea, two anthropophilic agroforestry species in order to conserve them sustainably. Thirty phytosociological surveys were sampled and the matrix gotten was subjected to an analysis of the adjusted correspondences (DCA) to discriminate the sacred forests with great diversity $(\mathrm{Rf}=130 ; \mathrm{H}=5.92 ; \mathrm{R}=0.97)$ of the fields/fallows of medium diversity $(\mathrm{Rf}=69 ; \mathrm{H}=3.40$; $\mathrm{R}=0.62$ ) and balanced. The average densities of stems went from 64,31 and 0 stems.ha $^{-1}$ in $D$. arborea to 30 ,
\end{abstract}


15 and 09 stems.ha $^{-1}$ in $N$. laevis in sacred forests and in fields/fallows from 28,16 and 0 stems.ha ${ }^{-1}$ in D. arborea at 89,56 and 13 stems.ha ${ }^{-1}$ in $N$. laevis respectively in guinean-congolese, sudano-guinean and sudanian zones. Phanerophytes constitute $85 \%$ of the average recovery in number and occupation of space. Guinean-congolese species are dominant and have a wide distribution spectrum. D. arborea and $N$. laevis keep better in sacred forests and fields / fallows respectively. The protection of potential habitats and the introduction of species into national reforestation programs as a priority would be an asset for better conservation.

(C) 2020 International Formulae Group. All rights reserved.

Keywords : Benin, Distribution, bioclimatic zone, Newbouldia laevis, Dracaena arborea.

\section{INTRODUCTION}

Le souci de conservation de la biodiversité, avec la prise en compte des besoins et aspirations des populations locales, est devenu réel depuis le Sommet de la Terre en 1992 (Kaou et al., 2017). Les espèces végétales sont très importantes pour les populations humaines en Afrique au Sud du Sahara en raison de leur contribution à la satisfaction des besoins en alimentation, santé, énergie, revenus et autres aspects du bien-être humain (Kaou et al., 2017; Gbesso et al., 2018), dans les traitements traditionnels des maladies qui impliquent des combinaisons de plantes médicinales et leurs utilisations socioculturelles et cultuelles (Dassekpo et al., 2017).

Le continent africain regorge de très grande diversité d'espèces végétales, dont la majorité est utilisée comme médicaments. En effet, sur les 300000 espèces végétales recensées au niveau de la planète, plus de 200000 espèces vivent dans les pays tropicaux d'Afrique et ont des vertus médicinales (Benlamdini et al., 2014). Au Bénin, sur 2807 espèces inventoriées dans les écosystèmes forestiers (Akoègninou et al., 2006), environ 1000 espèces sont utilisées comme plantes médicinales ou culturelles (Wedjangnon et al., 2016) dont $N$. laevis et $D$. arborea. L'Hysope africaine ou Newbouldia laevis (P. Beauv.) Seemann ex Bureau et Dracaena arborea (Willd.) Link ou le Dragonier africain sont deux essences agroforestières, pérennes et anthropophiles ayant peu de valeur économique, mais très sollicitée en pratiques traditionnelles et endogènes (Juhe-Beaulaton, 2009). Ces arbres sacrés se reconnaissent souvent par la présence d'un pagne blanc ou d'une ceinture de feuilles de palmier, accrochés sur leur tronc, de poteries où sont déposées des offrandes et l'existence dans leur voisinage de quelques plantes caractérisant les lieux de cultes (Dassekpo et al., 2017). Ces plantes seraient indicatrice d'anciens lieux visités par l'homme dans les forêts naturelles (JuheBeaulaton, 2009). Newbouldia laevis de la famille des Bignoniaceae est originaire d'Afrique tropicale et pousse dans les savanes guinéennes aux forêts denses, sur sol humide et bien sols drainés. Elle représente la seule espèce du genre Newbouldia rencontrée dans les forêts secondaires qui s'étendent du Sénégal au Cameroun, au Gabon, en République démocratique du Congo, en Angola (Usman \& Osuji, 2007). Au Bénin, Newbouldia laevis est retrouvée dans les forêts denses humides, dans les champs et jachères en zones Guinéo-congolaise et soudanoguinéenne et aussi régulièrement dans les habitations (Akoégninou et al., 2006). L'espèce est plus utilisée dans la délimitation des maisons (Adomou et al., 2018). L'Hysope renferme des propriétés antimicrobiennes (Fatounla et al., 2016), des flavonoïdes, des tannins, des terpènes, des glycosides stéroïdiens et des cardiotoniques. L'écorce contient des dérivés de l'harmane, harmine et harmol qui ont une action antihelmintique (Eyog Matig et al., 1999). Les activités de ces molécules bactéricides permettent l'utilisation de l'Hysope dans le traitement des douleurs abdominales, de la diarrhée, la dysenterie, contre les helminthiases (Mangambu et al., 2015), dans le déclenchement des contractions des parois utérines facilitant ainsi l'accouchement chez la femme enceinte (AkeAssi et al., 2010) et enfin dans le traitement de quelques maladies infantiles notamment le paludisme et la drépanocytose (Perumal et al., 2012). Les Dracaenaceae par contre, sont des 
monocotylédones renfermant les genres Dracaena et Sanseviera. Le genre Dracaena, regroupe 116 espèces dont 63 en Afrique tropicale. Le reste des espèces est partagé entre l'Asie, l'Australie, l'Amérique centrale et les régions chaudes de l'Europe (Masumbuko et al., 2013). D. arborea est rencontrée dans les habitations, les forêts denses humides, les forêts galeries, les forêts sacrées, les jardins de case, les jachères et dans les champs de huit (08) zones phytogéographiques sur les dix (10) du Bénin (Logbo et al., 2019). Des études phytochimiques sur les Dracaena ont révélé l'existence des di-terpènes, de saponines de type stéroïdique, consistant souvent en des glycosides de spirostanol et de furostanol; de génines triterpéniques, d'hétérosides et des métabolites secondaires. Diverses autres classes de composés caractérisées et quantifiées révèlent la présence des flavonoïdes du métacyclophane (Allabi et al., 2011), des stérols et des triterpénoïdes (Awono et al., 2003), des alcaloïdes, de l'oxalate de calcium, etc., qui ont des propriétés biologiques avérées entrant dans le traitement des affections telles que l'infertilité qui est une complication fréquente chez les hommes diabétiques, dans l'amélioration de la morphologie des testicules et dans la restauration de la spermatogenèse chez les diabétiques de type 1 sans avoir des propriétés anti-hyperglycémiques majeures (Salhi et al., 2010). Dracaena arborea est souvent utilisé en ethnomédecine grâce aux flavonoïdes qu'il renferme et qui possèdent des propriétés antioxydantes, anti-allergiques, anticancéreuses, anti-inflammatoires et antiviraux (Aniruddha et al., 2011). Par contre, les flavonoïdes jouent un rôle de défense chez les herbivores, dans la prise en charge du paludisme, le diabète et l'hypertension (Lenoble et al., 2001). Les alcaloïdes provenant de différents acides aminés ou acides mévaloniques conduisent au développement de médicaments anti-douleur chez l'homme (Chibembe et al., 2015). Dracaena arborea dispose à la fois des propriétés antibactériennes et antifongiques largement utilisées en cosmétique grâce aux molécules de saponines (Aniruddha et al., 2011), de l'oxalate de calcium, du phytate ou acide phytique (Inosytol hexapho-phosphate ou IP6) qui constitue la forme de stockage du phosphore dans de nombreux tissus végétaux. Le phosphore sous cette forme n'est pas biodisponible pour les humains, car ne contenant pas de la phytase qui l'enzyme digestive responsable de la séparation du phosphore de la molécule de phytate (Chibembe et al., 2015). Des études récentes ont montré que l'acide phytique pourrait avoir un effet préventif sur certains cancers comme ceux de la prostate, du sein, du pancréas et du colon (Flores et al., 2011). Au vu de tout ce qui précède, $N$. laevis et $D$. arborea constituent donc deux espèces végétales importantes pour les populations. Lawin et al. (2016), en s'intéressant à l'évaluation des menaces pesant sur les espèces et les stratégies endogènes de leur conservation ont attribué à $N$. laevis une menace de niveau 2 de pression sur une échelle de 3 niveaux de pression. Pour ces auteurs, les critères d'évaluation des menaces pour les organes recherchés et la distribution géographique et l'abondance de $N$. laevis sont chacun de niveau 3. Le nombre d'organes recherchés et les pratiques traditionnelles de conservation et de culture de l'espèce sont au niveau 2 chacun. L'importance de l'utilisation de l'espèce est au niveau 1. Des études ont été réalisées sur l'écologie des espèces (Adjanohoun et al., 1989; Akoegninou et al., 2006), sur leurs utilisations ethnobotaniques (Dassekpo et al., 2017 ; Adomou et al., 2018; Logbo et al., 2019) et enfin sur leur vulnérabilité et les stratégies endogènes de leurs conservations (Lawin et al., 2016). Le défi à relever dans un contexte où les modes d'utilisation des terres et l'urbanisation sont sans cesse croissants suite à l'accroissement démographique, est de renforcer la valorisation et la gestion durable de ces espèces anthropophiles à haute valeur socioculturelle. La présente étude a pour objectif général de déterminer la distribution et les habitats potentiels de $N$. laevis et de $D$. arborea, deux espèces anthropophiles dans les zones bioclimatiques du Bénin afin de disposer des données scientifiques pouvant permettre leur gestion durable dans le cadre de la valorisation des espèces d'intérêt pour les populations. 


\section{MATERIL ET METHODES Milieu d'étude}

Le Bénin est situé dans la zone intertropicale en Afrique de l'Ouest, entre les parallèles $6^{\circ} 10^{\prime}$ et $12^{\circ} 25^{\prime}$ Nord et les méridiens $0^{\circ} 45^{\prime}$ et $3^{\circ} 55^{\prime}$ Est (Figure 1). Il couvre une superficie de $114763 \mathrm{~km}^{2}$ avec une population 10008749 habitants pour une densité moyenne de 87,2 habitants au $\mathrm{km}^{2}$ (INSAE, 2015). Sur le plan climatique, le milieu d'étude couvre les trois zones bioclimatiques du pays (Adjanohoun et al., 1989). Il s'agit de la zone guinéo-congolaise dans le Sud $\left(6^{\circ} 25^{\prime}\right.$ à $\left.7^{\circ} 30^{\prime} \mathrm{N}\right)$, de la zone soudanienne au Nord $\left(9^{\circ} 45^{\prime}\right.$ à $\left.12^{\circ} 25^{\prime} \mathrm{N}\right)$ et la zone de transition soudano-guinéenne au centre $\left(7^{\circ} 30^{\prime}\right.$ à $\left.9^{\circ} 45^{\prime} \mathrm{N}\right)$. L'étude a été réalisée dans six (06) zones phytogéographiques sur les dix (10) que compte le Bénin et regroupant les départements de l'Atlantique, du Littoral, de l'Ouémé, du Plateau, du Mono, du Couffo, du Zou, des Collines, du Borgou, de l'Atacora et de la Donga. Ces zones phytogeographiques représentent des subdivisions des zones bioclimatiques suivant des considérations pédo-climatiques.

- la zone guinéo-congolaise est située entre $6^{\circ} 25^{\prime}$ et $7^{\circ} 30^{\prime} \mathrm{N}$ comporte quatre saisons. Le régime pluviométrique est bimodal (avriljuin et septembre-novembre) avec une moyenne annuelle de $1200 \mathrm{~mm}$ avec en moyenne 250 jours de pluies. Cette distribution devient unimodale entre mai et octobre à partir de $8^{\circ} \mathrm{N}$ avec plus de $900 \mathrm{~mm}$ de hauteur de pluie en moyenne. La différence de précipitation entre les mois les plus secs et les plus humides est de $167 \mathrm{~mm}$. La grande saison des pluies va de mars à fin juillet et la petite saison de septembre à mi-novembre (Figure 2A). Ces périodes pluvieuses sont alternées de deux saisons sèches : une grande, de minovembre à mi-mars et une petite d'août à septembre (Adjanohoun et al., 1989). La température moyenne journalière varie de 25 ${ }^{\circ} \mathrm{C}$ à $29^{\circ} \mathrm{C}(\mathrm{FAO}, 1998)$, la moyenne annuelle est de $26,8{ }^{\circ} \mathrm{C}$. Sur l'année, la variation de température est de $4,4{ }^{\circ} \mathrm{C}$. Le mois de février est le plus chaud de l'année avec une température moyenne de $29,0{ }^{\circ} \mathrm{C}$. Le mois d'août par contre, est le plus froid de l'année à une température moyenne de $24,6^{\circ} \mathrm{C}$.
L'humidité relative de l'air varie entre $69 \%$ et $97 \%$. Sur les plans pédologique et floristique, le Bénin est caractérisé par dix (10) phytodistricts (Adomou et al., 2006). Les phytodistricts de la zone guinéo-congolaise sont caractérisés par des fourrés et des mangroves sur des sols sableux, hydromorphes et halomorphes, de forêts semi décidues sur des sols ferralitiques et hydromorphes. Ces derniers sont également couverts de forêts marécageuses. Les sols sont ferralitiques profonds et peu fertiles $\left(7000 \mathrm{~km}^{2}\right)$, alluviaux et vertisols $\left(3600 \mathrm{~km}^{2}\right)$ et sont rencontrés dans les vallées des fleuves Mono, Niger, Couffo et Ouémé et dans la dépression de la Lama (Assogbadjo, 2006). Ces sols riches en argile, en matière organique et en éléments minéraux renferment d'une part des formations de sols bien drainés, composés de cordons sableux (anciens ou récents), des fourrés soit au stade avancé de colonisation des cordons, soit au stade de dégradation d'une ancienne forêt littorale et d'autre part, des formations des zones humides (lagunes et vasières) présentant deux types physionomiques fondamentaux : la forêt mangrove, constituée de palétuviers rouges (Rhizophora racemosa) et blanc (Avicenia germinans) longeant les lagunes jeunes et la forêt marécageuse à Anthocleista vogelii, à Raphia hookeri et à Alchornea cordifolia occupant les lagunes anciennes.

- la zone soudano-guinéenne est située entre $7^{\circ} 30^{\prime}$ et $9^{\circ} 45^{\prime} \mathrm{N}$ et dispose un régime pluviométrique de type uni-modal (MaiOctobre). La pluviométrie moyenne annuelle varie de $900 \mathrm{~mm}$ à $1116 \mathrm{~mm}$ répartie le plus souvent sur 113 jours en moyenne. Une différence de $190 \mathrm{~mm}$ est enregistrée entre le mois le plus sec et le mois le plus humide. L'humidité relative de l'air varie de $31 \%$ à $98 \%$. L'insolation moyenne s'élève à 2305 heures par an. Les températures varient entre $25{ }^{\circ} \mathrm{C}$ et $29{ }^{\circ} \mathrm{C}$. La température annuelle moyenne est de $26,9{ }^{\circ} \mathrm{C}$. L'amplitude thermique annuelle est de $4,5{ }^{\circ} \mathrm{C}$. Le mois de mars est le plus chaud de l'année avec une température moyenne de $29,0{ }^{\circ} \mathrm{C}$. Le mois d'août est le plus froid de l'année qui est de 24,5 ${ }^{\circ} \mathrm{C}$ (Figure 2B). Les trois phytodistricts de la zone de transition soudano-guinéenne sont constitués de forêts semi-décidues, de forêts 
claires et de forêts galeries sur des sols ferralitiques. Dans cette zone, les sols ferrugineux installés sur des roches cristallines accueillent des forêts sèches, des forêts claires et des forêts galeries. La zone soudanoguinéenne en prolongeant la zone à affinités guinéennes, constitue le domaine des mosaïques de forêts claires, avec des forêts denses sèches, parsemées de savanes arborées et arbustives et traversées par des galeries forestières (Adjanohoun et al., 1989). On trouve des sols à minéraux peu évolués et peu fertiles et des sols ferrugineux sur socle cristallin de fertilité variable (Assogbadjo, 2006). Les savanes arborées et arbustives composées de Vitellaria paradoxa, Adansonia digitata, Parkia biglobosa, Isoberlinia doka, Isoberlinia tomentosa, Combretum hypopilinum, Combretum sokodense, Combretum nigricans et Terminalia macroptera sont notées sur les sols hydromorphes; Isoberlinia doka et Detarium microcarpum sur les cuirasses ou roches peu profondes et enfin, Ceiba pentandra, Milicia excelsa et Antiaris africana sont observés dans les galeries forestières et îlots forestiers (Assogbadjo, 2006).

- La zone soudanienne est située entre $9^{\circ} 45^{\prime} \mathrm{N}$ et $12^{\circ} 25^{\prime} \mathrm{N}$ renferme une pluviométrie souvent inférieure à $900 \mathrm{~mm}$ par an répartie en moyenne sur 145 jours (Figure 2C). L'humidité de l'air varie de $18 \%$ pendant l'harmattan (décembre à février) à $99 \%$ en saison de pluies (août). Les températures moyennes mensuelles varient entre $24{ }^{\circ} \mathrm{C}$ et $31{ }^{\circ} \mathrm{C}$. Le nombre d'heures ensoleillées est de 2862 heures par an. Les sols sont hydromorphes, drainés renfermant par endroit des cuirasses ferralitiques et des lithosols. La végétation est constituée de savanes et de galeries forestières avec des arbres recouvrant faiblement le sol (Adjanohoun et al., 1989). Au sud, la végétation est analogue à celle de la zone de transition avec des peuplements de Isoberlina doka, de Isoberlina tomentosa, de Adansonia digitata, de Pterocarpus erinaceus, de Afzelia africana, de Erythrophleum guineense, de Ambligonocarpus andongensis et de Swartzia madagascariensis. La végétation herbacée est dense dans les parties arbustives avec des espèces comme Cymbopogon giganteus et
Lantana rhodesiensis. Au nord, la taille des espèces ligneuses diminue, suivi d'un changement dans la composition floristique. Des espèces de savane telles que Combretum micranthum, Combretum glutinosum, Combretum nigricans et Boscia salicifolia sont observées (Assogbadjo, 2006).

\section{Matériel végétal}

Le matériel végétal utilisé était constitué des arbres de Newbouldia laevis et de Dracaena arborea rencontrés dans les communes abritant le milieu d'étude.

\section{Description botanique de Dracaena arborea et de Newbouldia laevis}

- Dracaena arborea est de la famille des Dracaenaceae qui regroupe 116 espèces dont 63 en Afrique tropicale. Le reste des espèces étant partagé dans les zones chaudes d'Amérique, d'Australie, d'Asie et d'Europe (Ilodibia et al., 2015). Sa présence dans les forêts naturelles est aussi indicatrice d'anciens lieux visités par l'homme (Juhe-Beaulaton, 2009). C'est une plante à port arboré ou arbustif 2 à $15 \mathrm{~m}$ de hauteur au stade adulte. Les feuilles de forme linéaire-lancéolée sont longues de 40 à $125 \mathrm{~cm}$ et larges de 5 à $10 \mathrm{~cm}$. Les inflorescences sont simples ou paniculées de 15 à $125 \mathrm{~cm}$ de long. Les fleurs environ au nombre de 20 ou plus sont de couleur blanche, bariolées de rose. Les fleurs sont arrangées en glomérules terminales et axillaires. La floraison se déroule entre août et septembre donnant des fruits de couleur orange à maturité (Farnsworth et al., 1999).

- Newbouldia laevis est à port arbustif de 3 à 25 $\mathrm{m}$ de hauteur. Les feuilles sont composées imparipennées avec 3 à 6 paires de folioles à bords dentés. Les feuilles sont longues de 20 $\mathrm{cm}$ et larges de $10 \mathrm{~cm}$ (Akoègninou et al., 2006). L'inflorescence est une panicule terminale constituée de grandes fleurs de couleur rose-blanchâtre. La floraison a lieu de décembre à janvier et la fructification se produit en mars. Les fruits sont des siliques de $25 \mathrm{~cm}$ de longueur portant des valves trinervées contenant des graines ailées de $3 \mathrm{~cm}$ de longueur (Akouègninou et al., 2006). L'Hysope se reproduit par les graines. Le mode de reproduction le plus utilisé est le bouturage 
car il est beaucoup plus rapide (Adomou et al., 2018). L'Hysope est rencontrée dans les reliques de forêts denses, les champs, les jachères et les jardins de cases et est utilisée comme des poteaux de clôture en milieu rural. $N$. laevis est une espèce de la zone guinéenne et peut s'établir et s'adapter à divers types de climats.

\section{Méthodes d'étude}

Les points d'occurrence matérialisant la présence des espèces sur le terrain ont été obtenus à l'aide d'un GPS lors de l'enquête exploratoire effectuée dans les trois zones bioclimatiques sur la disponibilité des espèces, puis matérialisés sur les cartes. Ces points de présence des espèces ont servi dans le choix des communes constituant le milieu d'étude.

\section{Outils de collecte des données}

Le matériel de collecte des données utilisé était constitué d'un ruban pour la délimitation des placeaux, de bandes fluorescentes pour la matérialisation des limites des placeaux, de coupe-coupe pour l'ouverture des layons et la confection des piquets de coins, de sécateur pour le prélèvement des échantillons, du ruban $\mathrm{R}$ pour la mesure des diamètres des arbres à $1,30 \mathrm{~m}$ audessus du sol, du clinomètre SUNTO pour la mesure des azimuts, d'un GPS (Global Positionning System) pour le géoréférencement des pieds des $N$. laevis et de $D$. arborea, des sites échantillonnés, des fiches de relevés de végétation et une carte de situation géographique des secteurs d'étude.

\section{Collecte des données}

Les relevés phytosociologiques ont été effectués suivant la méthode sigmatiste de Braun-Blanquet. L'aire de relevé retenue est basée sur les travaux de Djego et al. (2006) effectués en milieu tropical et qui utilisent des surfaces variant entre $100 \mathrm{~m}^{2}$ et $1000 \mathrm{~m}^{2}$ selon les formations végétales et les strates. Dans la présente étude, l'aire de relevé était de $100 \mathrm{~m}^{2}$ $(10 \mathrm{~m} \times 10 \mathrm{~m})$ pour la strate herbacée et de 900 $\mathrm{m}^{2}(30 \mathrm{~m} \times 30 \mathrm{~m})$ pour les strates arbustive et arborée. L'identification des types biologiques des espèces a été réalisée sur la base des travaux de Raunkiaer (1934). Les types phytogéographiques des espèces ont été établis à partir des subdivisions chorologiques de White (1986). Les données collectées ont porté sur la distribution spatiale et la densité des tiges à l'hectare et sur la caractérisation phytoécologique des communautés végétales hébergeant $N$. laevis et de $D$. arborea. La caractérisation des habitats de $N$. laevis et de $D$. arborea a été effectuée dans les trois zones bioclimatiques. Les coordonnées géographiques des localités de présence des espèces ont été enregistrées à l'échelle des zones phytogéographiques et aussi des zones bioclimatiques. Le nombre de placeaux et de transects par formation végétale a été déterminé sur la base de l'étendue des formations végétales et ont été installés en tenant compte de l'homogénéité floristique et la topographique des stations. Cinq (05) placeaux et 01 transect ont été réalisés par phytodistrict. Un total de 30 placeaux et 06 transects à bande de $15 \mathrm{~m}$ de large et de 3 kilomètres de long pour l'ensemble des six (06) phytodistricts a été installé à travers les différentes formations végétales (forêts sacrées, champs/jachères). Les placeaux ont été installés de façon aléatoire avec une équidistance de $200 \mathrm{~m}$ dans les strates de champs/jachères et de $50 \mathrm{~m}$ en milieu naturelle dans les strates de forêts sacrées. Les espèces végétales rencontrées sur le terrain ont été identifiées et celles non identifiées ont été échantillonnées puis déterminées à l'Herbier National du Bénin par comparaison aux spécimens de référence.

\section{Traitement et analyses des données}

Le tableau de contingence en présenceabsence des espèces a été établi avec le tableur Excel à partir des données de relevés floristiques. Les données obtenues ont été soumises à une analyse des correspondances redressées (DCA) pour discriminer les groupements végétaux. Les espèces indicatrices ont été utilisées pour nommer les groupements végétaux. Les paramètres tels que la richesse spécifique des groupements végétaux, les indices de diversité de ShannonWeaver et de l'équitabilité de Pielou (E) ont été calculés pour caractériser les groupements végétaux. 
- La densité du peuplement (N) correspond au nombre de tiges à l'hectare. Elle est obtenue par la formule :

$$
\boldsymbol{N}=\frac{n}{\mathrm{~s}}
$$

Où $\mathrm{n}$ est le nombre total d'individus d'arbres inventoriés dans le groupe de relevé et S l'aire totale échantillonnée dans le groupe de relevé en hectare ;

- l'indice de diversité de Shannon-Wiener $(\mathrm{H})$ a permis de mesurer la diversité spécifique au niveau d'un placeau. Il est donné par la formule

$$
H=-\sum P i * \log _{2} P i
$$

$\mathrm{H}=$ indice de diversité de ShannonWiener. Il s'exprime en bits. Si H $€$ ]0 ; 3 [, alors $\mathrm{H}$ est supposé faible (cas des stations spécialisées où il s'agit généralement des phénomènes de dominance d'une espèce ou d'un petit nombre d'espèces sur l'ensemble des espèces de la communauté). Si H $€[3 ; 4[$, alors $\mathrm{H}$ est supposé moyen et enfin si $\mathrm{H} \in[4 ;+[$, alors H est supposé élevé (cas des stations isotropes où les conditions sont très favorables à un grand nombre d'espèces dans des proportions quasi-égales).

- l'indice d'équitabilité de Pielou (E) est la mesure du degré de diversité atteint par un groupement végétal. Il traduit la manière dont les individus sont distribués à travers les groupements végétaux. Il varie entre 0 et 1 . L'indice d'équitabilité de Pielou $\mathrm{E}<0,5$, lorsqu'il y a un phénomène de dominance (les individus appartiennent à une seule espèce). L'indice d'équitabilité de Pielou $\mathrm{E} \geq 0,5$, lorsque la répartition des individus est homogène et se traduit de façon équitable. Les espèces se répartissent de façon régulière et que les individus ont exactement le même recouvrement. L'indice d'équitabilité de Pielou (E) se calcule par la formule suivante :

$E=\frac{H}{\log _{2}(R s)}$

Où Rs désigne la richesse spécifique.
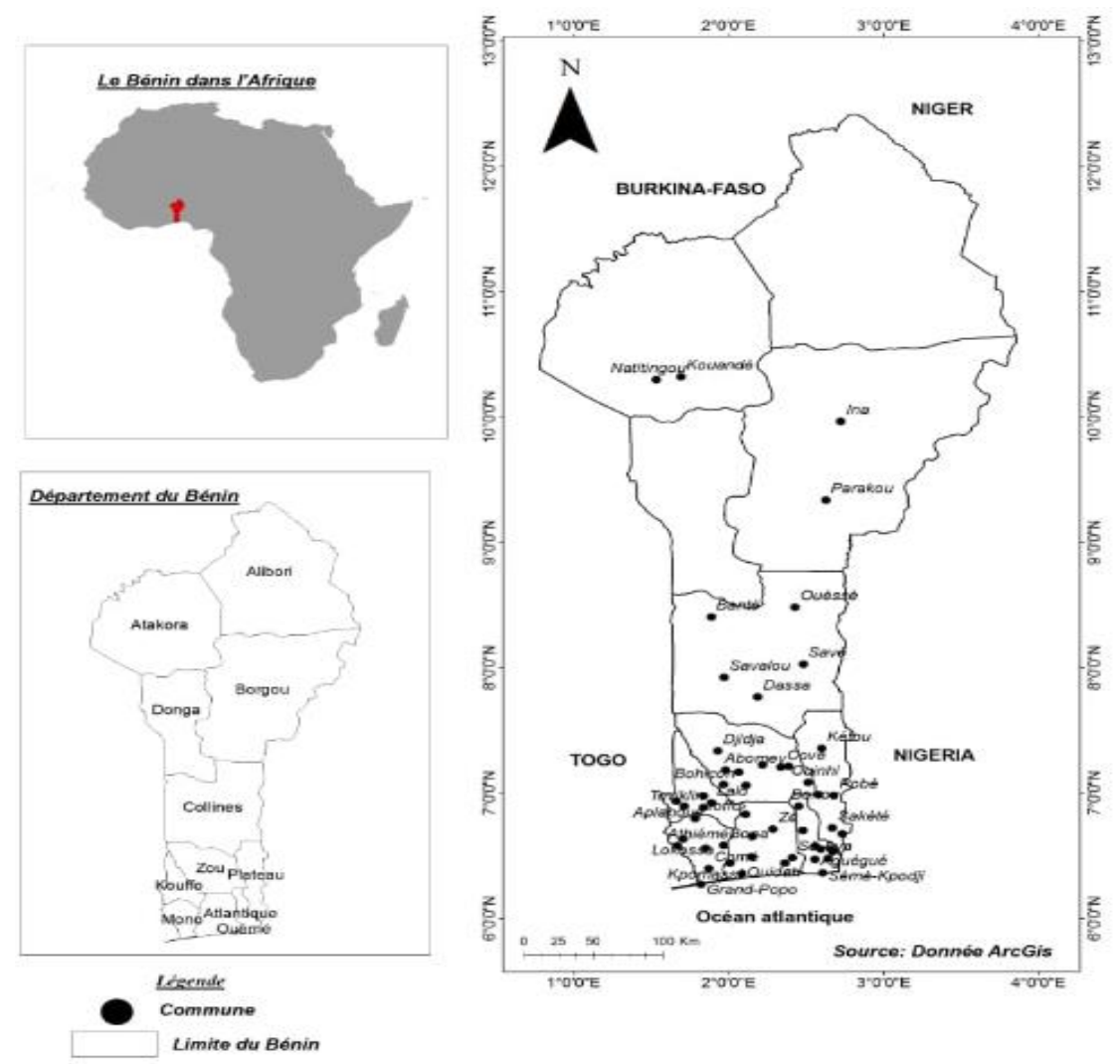

Figure 1 : Carte de localisation des milieux d'étude. 

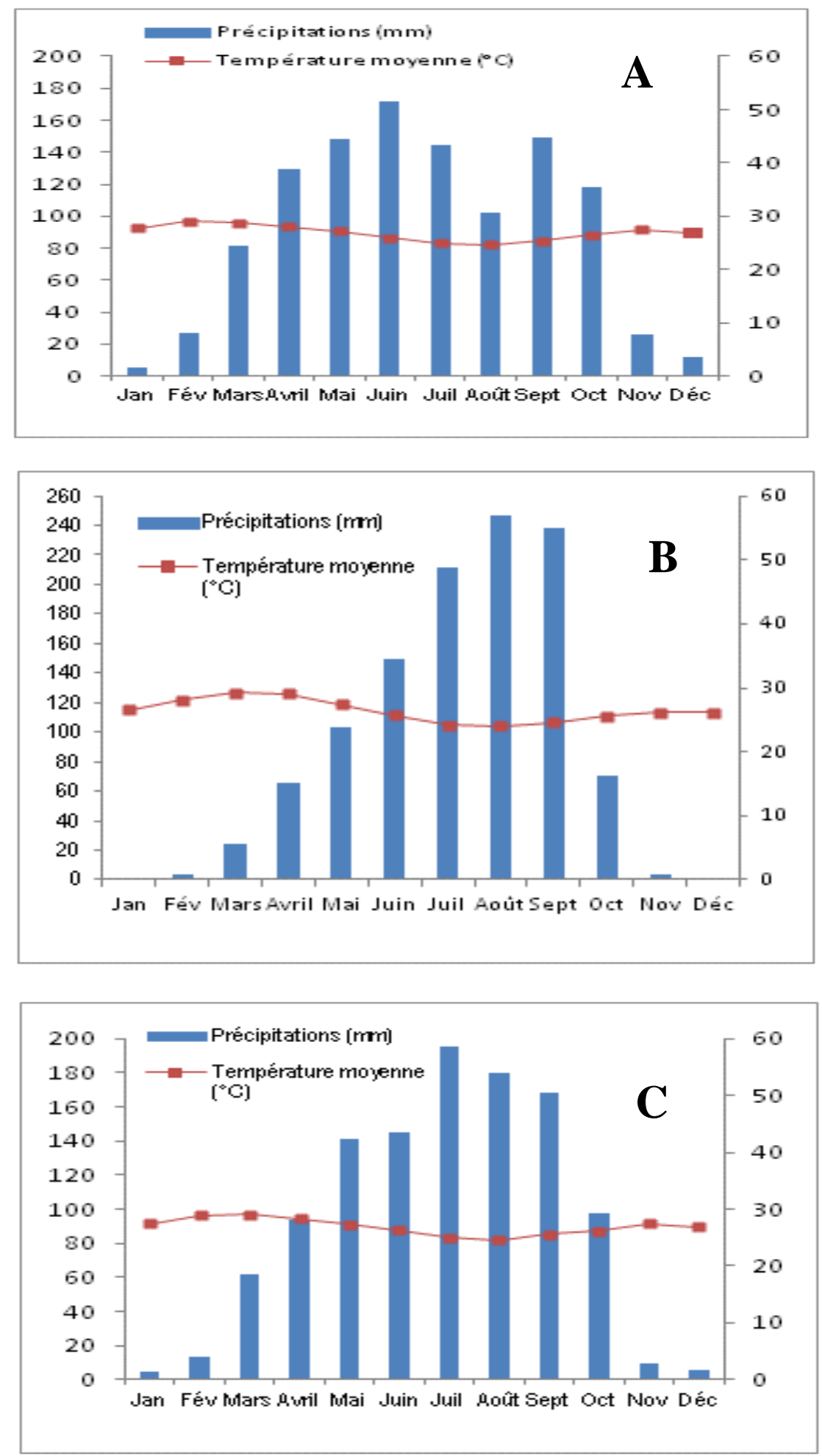

Figure 2: Diagrammes ombrothermiques des zones guinéo-congolaise (2A), soudano-guinéenne (2B) et soudanienne $(2 \mathrm{C})$. 


\section{RESULTATS \\ Points d'occurrence de $N$. laevis et $D$. arborea}

La distribution des points d'occurrence de $N$. laevis se fait suivant les trois zones bioclimatiques et ayant pour barycentre et centre de distribution la zone guineocongolaise (Figure 3A). Les pourcentages de distribution des points d'occurrence sont de $84 \%, 15 \%$ et $1 \%$ respectivement en zone guineo-congolaise, soudano-guinéenne et soudanienne (Figure 3B) et regroupent les phytodistricts du côtier, du Plateau, de la vallée de l'Ouémé, de Pobè, du Zou, de Bassila, du Borgou sud, de la chaîne de l'Atacora et du Borgou nord (Figure 4A). Les valeurs maximales des pourcentages des points d'occurrences sont variables et atteignent 42\% dans le phytodistrict du Plateau, 20\% dans celui de Pobè, $10 \%$ dans les phytodistricts de la vallée de l'Ouémé, du Côtier et du Zou, 3\% dans les phytodistricts de Bassila et Borgou sud et enfin $1 \%$ dans les phytodistricts de Borgou nord et de la chaîne de l'Atacora (Figure 4B).

Chez D. arborea, la distribution des points d'occurrence sont observés dans les zones guinéenne et soudano-guinéenne et avec pour barycentre et centre de distribution la zone guinéenne (Figure 5A). Les valeurs maximales des pourcentages des points d'occurrence sont de $83 \%, 17 \%$ et $0 \%$ respectivement en zones guinéo-congolaise, soudano-guinéenne et soudanienne (Figure 5B). Ces points d'occurrences se répartissent dans les phytodistricts du côtier, du Plateau, de la vallée de l'Ouémé, de Pobè, du Zou et de Bassila ; soit six (06) phytodistricts sur les dix (10) (Figure 6A). Les valeurs maximales des pourcentages des points d'occurrences sont variables et peuvent atteindre $48 \%$ dans le phytodistrict du Plateau, 15\% à Pobè, 15\% dans le Zou, $10 \%$ dans le Côtier, $10 \%$ dans la vallée de l'Ouémé et enfin $2 \%$ à Bassila (Figure 6B).

\section{Individualisation et caractérisation des groupements végétaux}

L'analyse des correspondances (DCA) sur la matrice de donnée du tableau phytosociologique, montre que les deux premiers axes du diagramme euclidien cumulent $29,8 \%$ de l'inertie totale et montrent ainsi une forte dispersion de l'information au niveau des axes factoriels. La carte factorielle des 30 relevés montre que l'axe 1 mesure le degré d'anthropisation et de dégradation de la végétation et l'axe 2 est celui de la discrimination de la structure de la végétation (Figure 7). Globalement, il ressort que les relevés effectués se répartissent en deux groupements végétaux. Le groupement végétal $\mathrm{G}_{1}$ composé de 10 relevés ( $\mathrm{SPFSB}_{4}, \mathrm{SPFSB}_{2}$, $\mathrm{SPFSZ}_{1}, \mathrm{SPFSB}_{1}, \mathrm{SPFSB}_{3}, \mathrm{SPFSB}_{5}, \mathrm{SPFSZ}_{3}$, $\mathrm{SPFSZ}_{2}, \quad \mathrm{SPFSZ}_{4}, \mathrm{SPFSZ}_{5}$, renferme les formations végétales à Albizia zygia et Triplochiton scleroxylon, Urera obovata \& Mucuna prurens, caractéristique des forêts sacrées (Figure 8). Par contre, le groupement végétal $\mathrm{G}_{2}$ est constitué de 20 relevés $\left(\mathrm{SPCJZ}_{2}\right.$, $\mathrm{SPCJZ}_{5}, \mathrm{SPCJZ}_{7}, \mathrm{SPCJZ}_{1}, \mathrm{SPCJZ}_{4}, \mathrm{SPCJZ}_{6}$, $\mathrm{SPCJB}_{2}, \mathrm{SPCJB}_{3}, \mathrm{SPCJB}_{7}, \mathrm{SPCJZ}_{8}, \mathrm{SPCJZ}_{10}$, $\mathrm{SPCJZ}_{3}, \mathrm{SPCJB}_{10}, \mathrm{SPCJZ}_{9}, \mathrm{SPCJB}_{6}, \mathrm{SPCJB}_{8}$, $\mathrm{SPCJB}_{1}, \mathrm{SPCJB}_{9}, \mathrm{SPCJB}_{4}$ et $\mathrm{SPCJB}_{5}$ ), et renferme les formations végétales à Mallotus oppositifolius et Newbouldia laevis, Mucuna prurens et Waltheria indica et est caractéristique des champs/jachères (Figure 8).

\section{Caractérisation écologique des groupements végétaux}

Les inventaires floristiques ont permis d'obtenir dans l'ensemble des groupements végétaux, une richesse spécifique (Rs) de cent quatre-vingt-dix-neuf (199) espèces, réparties en 116 genres et 74 familles (Tableau 1). Le groupement végétal $\mathrm{G}_{1}$ renferme la plus grande richesse spécifique avec 74 genres, 46 familles et 130 espèces. Le groupement végétal $\mathrm{G}_{2}$, composé de 42 genres, 28 familles et 69 espèces dispose de la plus faible richesse spécifique. Les Leguminoseae et les Euphorbiaceae constituent les familles dominantes, avec une proportion de $48 \%$ dont $63 \%$ de dicotylédones pour le groupement végétal G1. Par contre, le groupement végétal G2 renferme $22 \%$ dont $51 \%$ de dicotylédones. Les richesses spécifiques des deux espèces varient d'un groupement végétal à un autre et suivant les zones bioclimatques. 
En zone guinéo-congolaise, chez $D$. arborea, le groupement végétal $\mathrm{G}_{1}$ présente des indices de diversité de Shannon-Wiener et d'équitabilité de Pielou ( $\mathrm{H}=5,01$ bits; $\mathrm{E}=0,91)$ élevés par rapport au groupement végétal $\mathrm{G}_{2}$ $(\mathrm{H}=3,62$ bits; $\mathrm{E}=0,39)$. La comparaison entre les valeurs des indices montre que la diversité au sein du groupement végétal $\mathrm{G}_{1}$ est élevée et qu'il existe une équi-répartition des recouvrements entre des espèces plus nombreuses que dans le groupement végétal $\mathrm{G}_{2}$ (Tableau 2). Chez N. laevis, le groupement végétal $\mathrm{G}_{2}$ présente les indices de diversité de Shannon Shannon-Wiener et d'équitabilité de Pielou ( $\mathrm{H}=5,83$ bits ; $\mathrm{E}=0,94)$ plus élevés que dans le groupement végétal $\mathrm{G}_{1}(\mathrm{H}=3,84$ bits; $\mathrm{E}=0,42$ ). La comparaison des indices de diversité montre que les espèces constituant le groupement végétal $\mathrm{G}_{2}$ dont $N$. laevis sont plus diversifiées que dans le groupement végétal $\mathrm{G}_{1}$ (Tableau 2).

En zone soudano-guinéenne, le groupement végétal $\mathrm{G}_{1}$ abritant $D$. arborea (Tableau 2) présente des indices de diversité de Shannon-Wiener et d'équitabilité de Pielou $(\mathrm{H}=5,50$ bits ; $\mathrm{E}=0,89)$. Dans le groupement végétal $\mathrm{G}_{2}, D$. arborea est absente. Les indices de diversité de Shannon-Wiener et d'équitabilité de Pielou sont moyennes $(\mathrm{H}=3,05$ bits ; $\mathrm{E}=0,31)$ par rapport à $\mathrm{G}_{1}$. Les valeurs des indices montrent que le groupement végétal $\mathrm{G}_{1}$ dont $D$. arborea sont plus diversifiées et plus équilibrées les unes des autres. Chez N. laevis, le groupement végétal $\mathrm{G}_{2}$ présente des indices de diversité de Shannon-Wiener et d'équitabilité de Pielou $(\mathrm{H}=5,56$ bits; $\mathrm{E}=0,91)$ élevés par rapport à ceux $\mathrm{du}$ groupement végétal $\mathrm{G}_{1} \quad(\mathrm{H}=3,15$ bits; $\mathrm{E}=0,36$ ) qui sont moyennes. Le groupement végétal $\mathrm{G}_{2}$ renferme des espèces dont $N$. laevis qui sont plus diversifiées et plus équiprobables que dans le groupement végétal $\mathrm{G}_{1}$ (Tableau 2).

En Zone soudanienne, les groupements végétaux de forêts sacrées (G1) et de champs/jachères $(\mathrm{G} 2)$ ne renferment pas des plants de D. arborea. Par contre, N. laevis est rencontré dans les deux groupements végétaux avec de faibles indices de diversité de Shannon-Wiener et d'équitabilité de Pielou (Tableau 2).

\section{Densité moyenne de tiges à l'hectare de $D$.} arborea et $N$. laevis

Les densités moyennes des tiges des espèces en zones guinéo-congolaise, soudanoguinéenne et soudanienne avec les valeurs des erreurs types varient d'un groupement végétal à un autre et d'une zone bioclimatique à une autre (Tableau 3). En zone guinéo-congolaise, les densités moyennes des tiges sont élevées dans les groupements végétaux $\mathrm{G}_{1}$ chez $D$. arborea. Les valeurs moyennes des tiges à l'hectare passent de 64 tiges.ha $^{-1}$ dans le groupement végétale $\mathrm{G} 1$ à 31 tiges.ha $^{-1}$ dans la même formation végétale en zone soudanoguinéenne ; soit deux fois plus importantes. La même observation est faite dans la formation végétale $\mathrm{G}_{2}$ hébergeant $N$. laevis. Les densités moyennes de tige passent de 37 tiges.ha $^{-1}$ en zone guinéo congolaise à 11 tiges.ha $^{-1}$ en zone soudano guinéenne; soit une densité moyenne de tige à l'hectare trois fois plus importante et cinq fois plus importante lorsqu'on passe en zone soudanienne.

Dans le groupement végétal $\mathrm{G}_{2}$, les valeurs des densités moyennes de tiges chez $D$. arborea sont relativement faibles et diminuent lorsqu'on passe de la zone guinéo-congolaise à la zone soudano-guinéenne (tableau 3). Les mêmes observations sont faites chez $N$. laevis mais avec des valeurs de densité moyenne de tige relativement élevées. Les densités moyennes des tiges de $D$. arborea sont plus importantes dans le groupement végétal $\mathrm{G}_{1}$. L'espèce est totalement absente dans le groupement végétal $\mathrm{G}_{2}$. Par contre, les densités moyennes des tiges les plus importantes sont notées chez $N$. laevis dans le groupement végétal $\mathrm{G}_{2}$. En zone soudanienne, la densité de tiges est deux fois plus importante dans le groupement végétal G2 que dans celui de G1.

\section{Types biologiques et phytogéographiques des groupements végétaux}

Les analyses des spectres bruts et pondérés des groupements végétaux $G_{1}$ et $G_{2}$ après recensement des types biologique et phytogéographique montrent que les phanérophytes $(\mathrm{Ph})$ constituent les formes de vie dominantes $(84,68 \%)$ dans le groupement végétal $G_{1}$ (Figure 9A). Ensuite, viennent les 
thérophytes $(4,5 \%)$, les géophytes $(4,5 \%)$, les chaméphytes $(3,6 \%)$ et les hémicryptophytes $(2,7 \%)$. $\mathrm{Au}$ niveau des types phytogéographiques (Figure 9B), les spectres bruts montrent une forte dominance des espèces guinéo-congolaises $(30,92 \%)$ en terme de fréquence et d'abondance. Elles sont suivies des espèces soudano-guinéennes $(19,58 \%)$, paléotropicales $(15,46 \%), \quad$ soudaniennes $(13,40 \%)$, pantropicales $(9,27 \%)$, soudanozambéziennes $(9,27 \%)$, afroaméricaines et afrotropicales. Par contre, en considérant les spectres pondérés, on note également une forte abondance des espèces guinéo-congolaises $(44,43 \%)$, ensuite viennent les espèces soudaniennes $(17,49 \%)$, soudano-guinéennes $(14,64 \%)$, paléotropicales $(13,72 \%)$, soudanozambézienne $(6,23 \%)$, pantropicales (2\%), afroaméricaines et afrotropicales. Dans le groupement végétal $\mathrm{G}_{2}$ (Figure $10 \mathrm{~A}$ ), les spectres pondérés montrent que les phanérophytes $(72,05 \%)$ constituent la forme de vie la plus dominante. Suivent ensuite les thérophytes $(19,13 \%)$, les hémicryptophytes $(5,18 \%)$, les chaméphytes $(2 \%)$ et les géophytes $(1,64 \%)$. Les spectres bruts des types biologiques par contre, montrent que les phanérophytes représentent $80 \%$ des espèces, ensuite viennent les thérophytes (15\%), les hémicryptophytes $(2,5 \%)$, les chaméphytes $(1,5 \%)$ et les géophytes $(1 \%)$. Au niveau des types phytogéographiques (Figure 10B), les spectres bruts montrent que les espèces pantropicales $(34,21 \%)$ constituent les espèces les plus dominantes ensuite viennent les espèces guinéo-congolaises (21,05\%), paléotropicales $(17,10 \%)$, soudano-guinéennes $(11,84 \%), \quad$ soudaniennes $\quad(7,89 \%)$, afrotropicales $(5,26 \%)$ et soudanozambéziennes $(2,65 \%)$. Les spectres pondérés présentent une forte prédominance des espèces pantropicales $(58,04 \%)$. Suivent les espèces guinéo-congolaises $(23,43 \%)$, paléotropicales $(6,37 \%) \quad$ soudano-guinéennes $(6,21 \%)$, soudaniennes $(5,23 \%)$, afrotropicales $(0,40 \%)$ et soudano-zambéziennes $(0,32 \%)$.
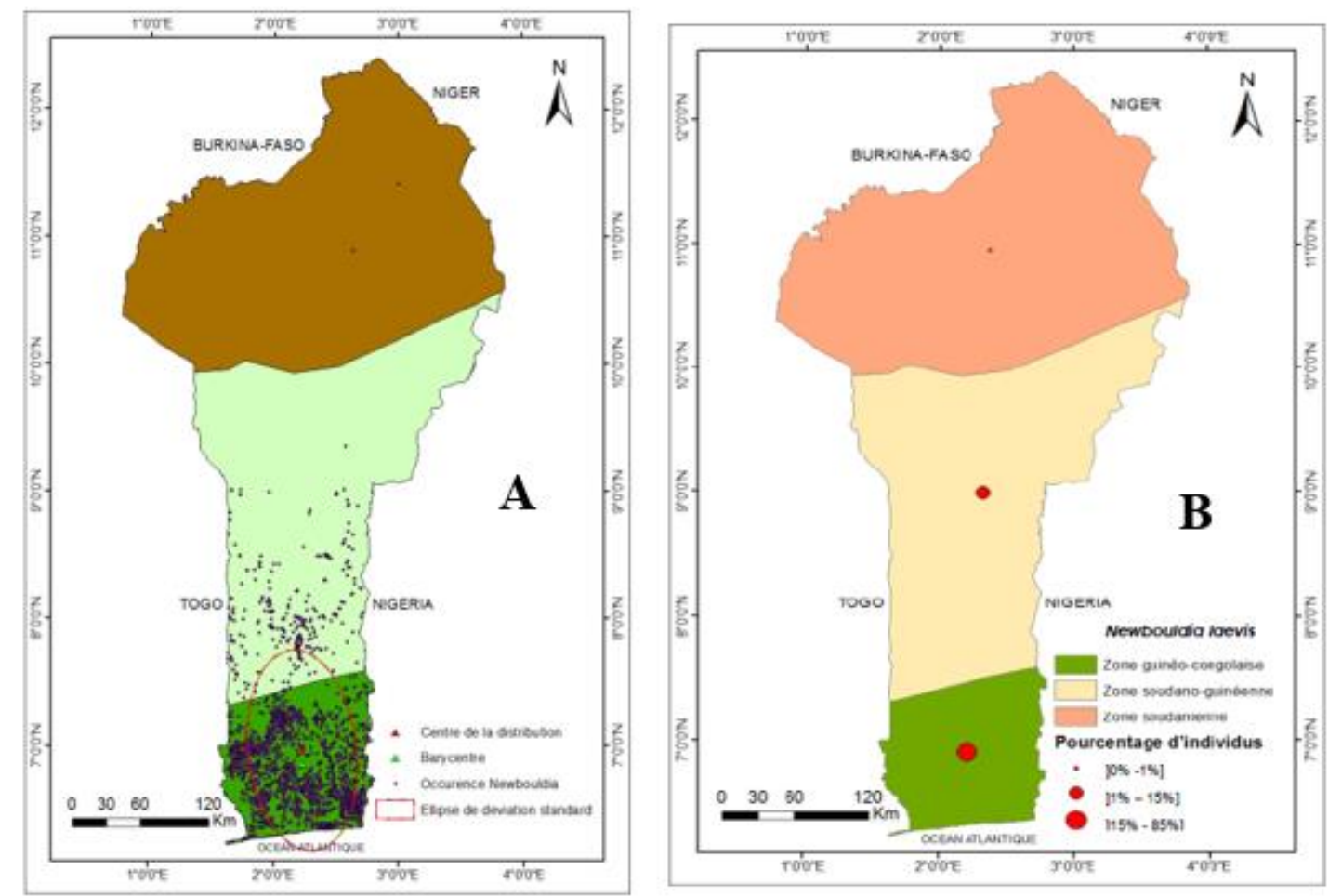

Figure 3 : Distribution (3A) et densité (3B) des points d'occurrence de Newbouldia. laevis dans les zones bioclimatiques. 


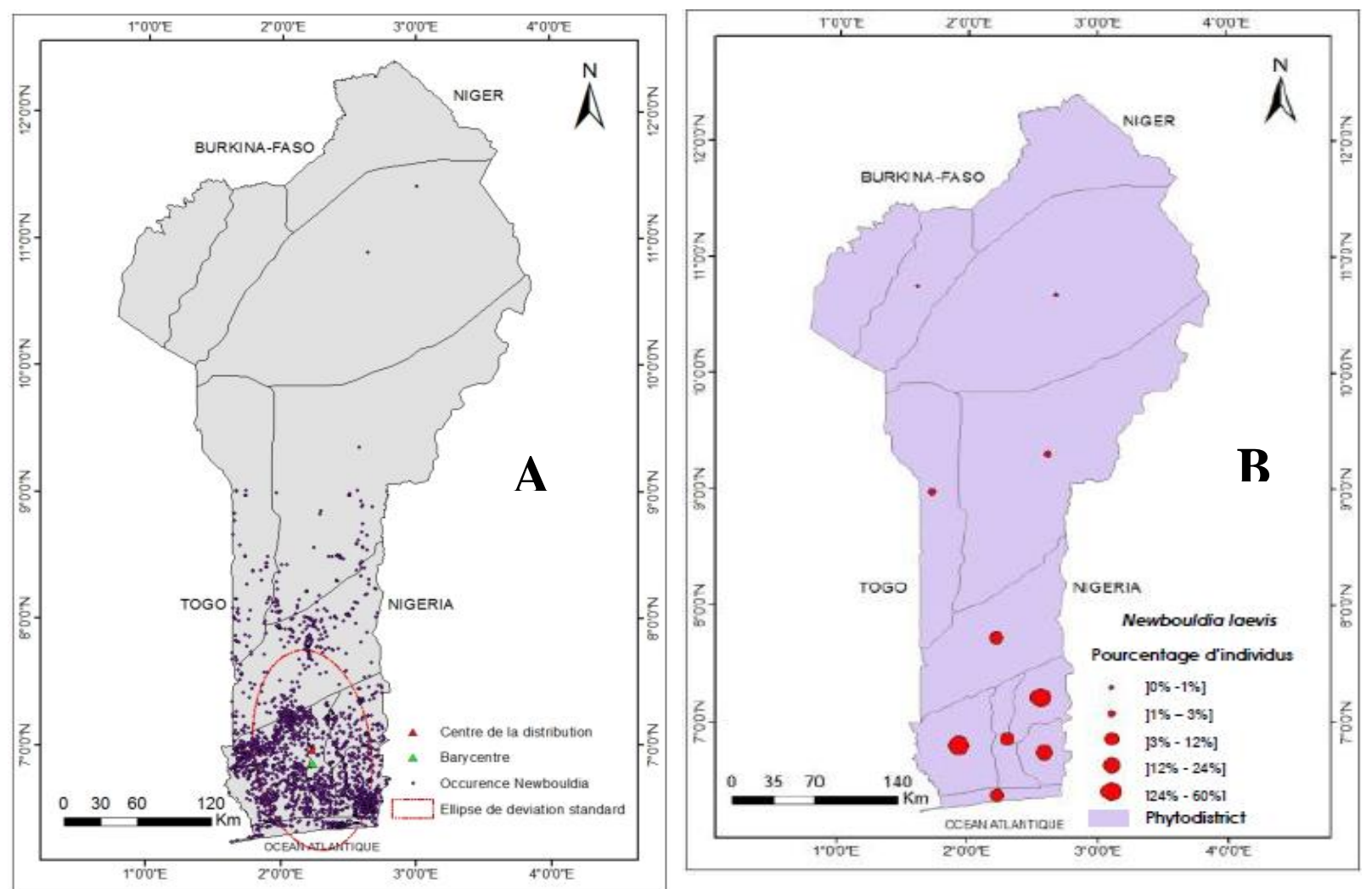

Figure 4 : Distribution (4A) et densité (4B) des points d'occurrence de Newbouldia. laevis dans les phytodistricts.
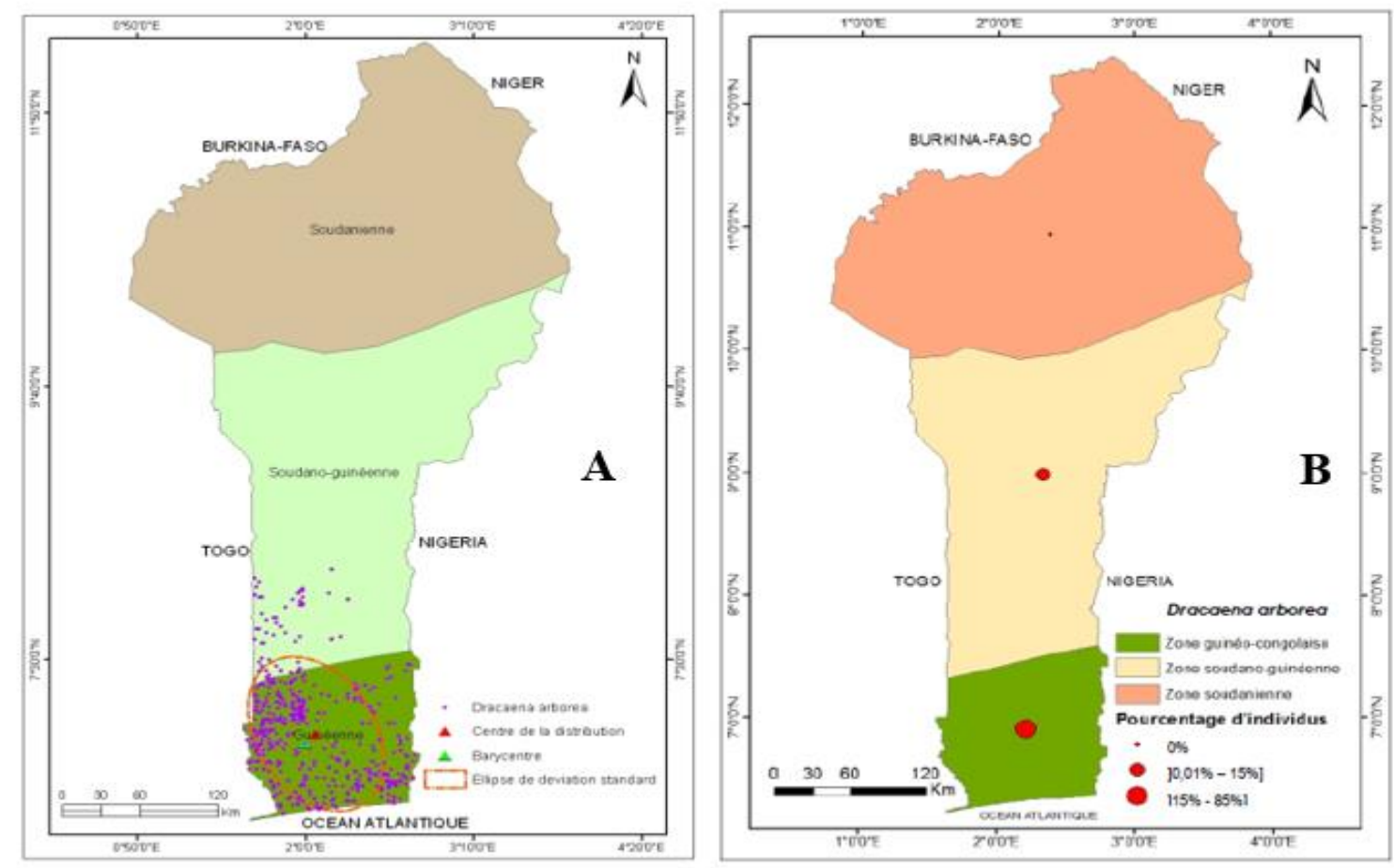

Figure 5 : Distribution (5A) et densité (5B) des points d'occurrence de Dracaena arborea dans les zones bioclimatiques. 

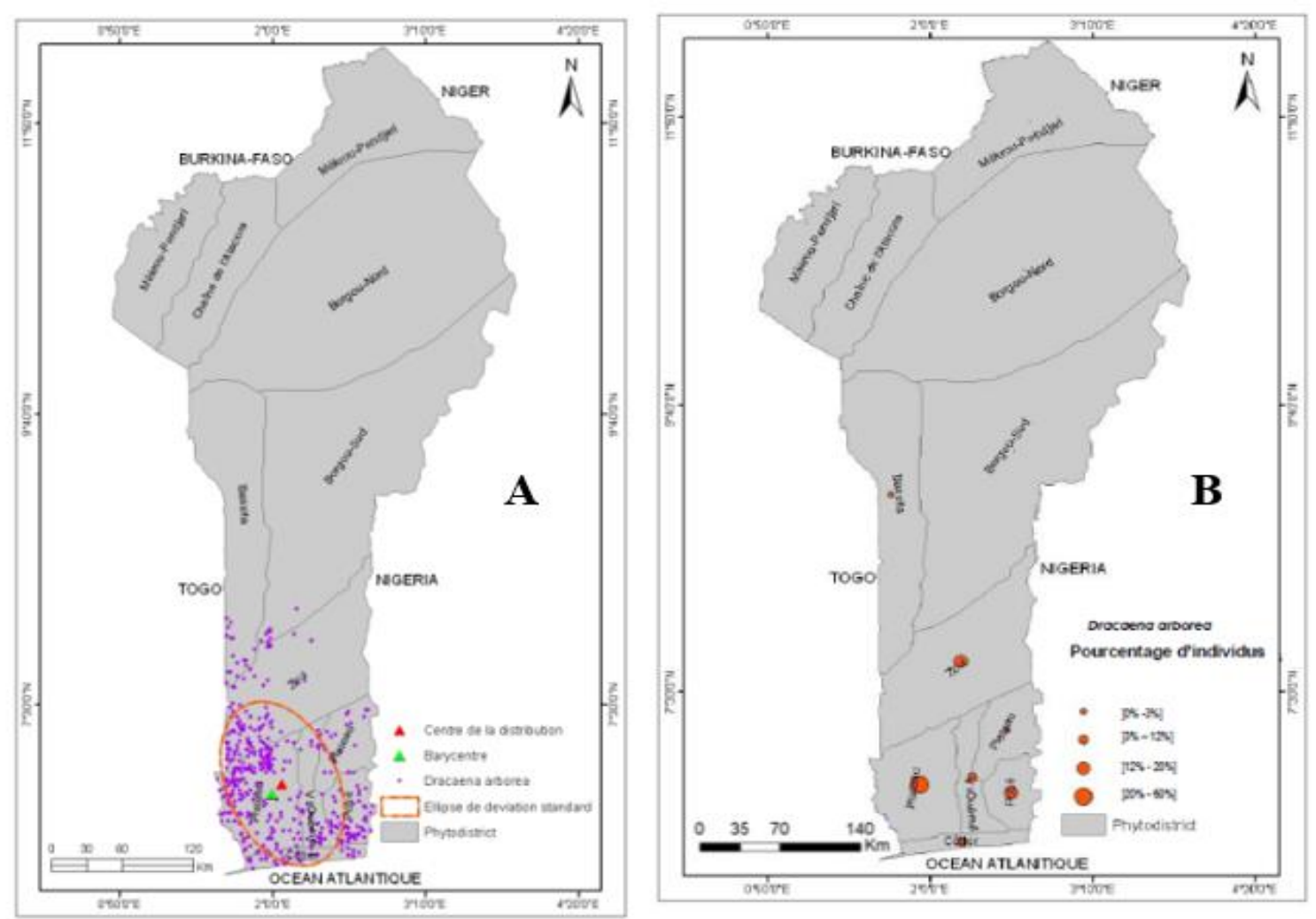

Figure 6 : Distribution (6A) et densité (6B) des points d'occurrence de Dracaena arborea dans les phytodistricts.

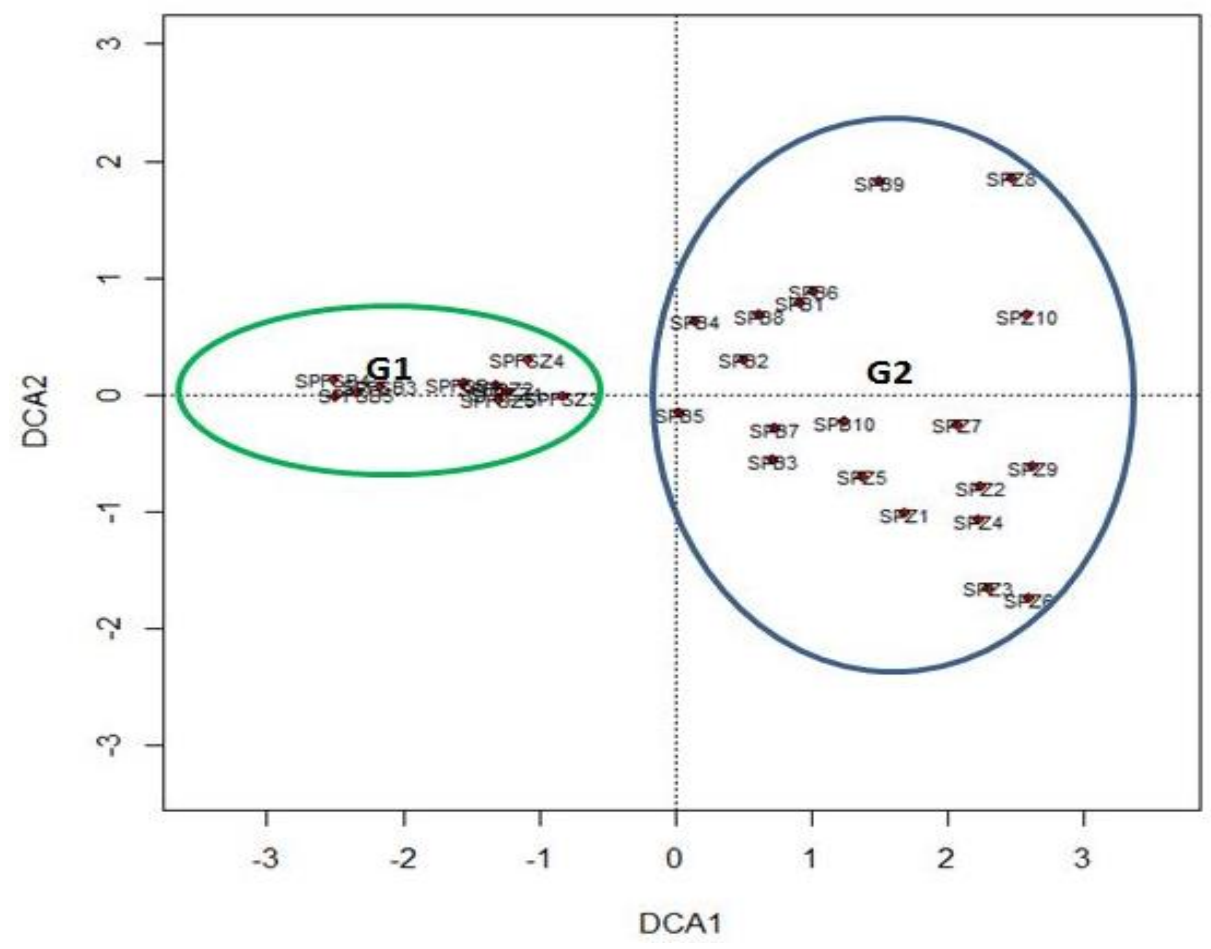

Figure 7 : Répartition des relevés dans les plans factoriels des axes 1 et 2. 


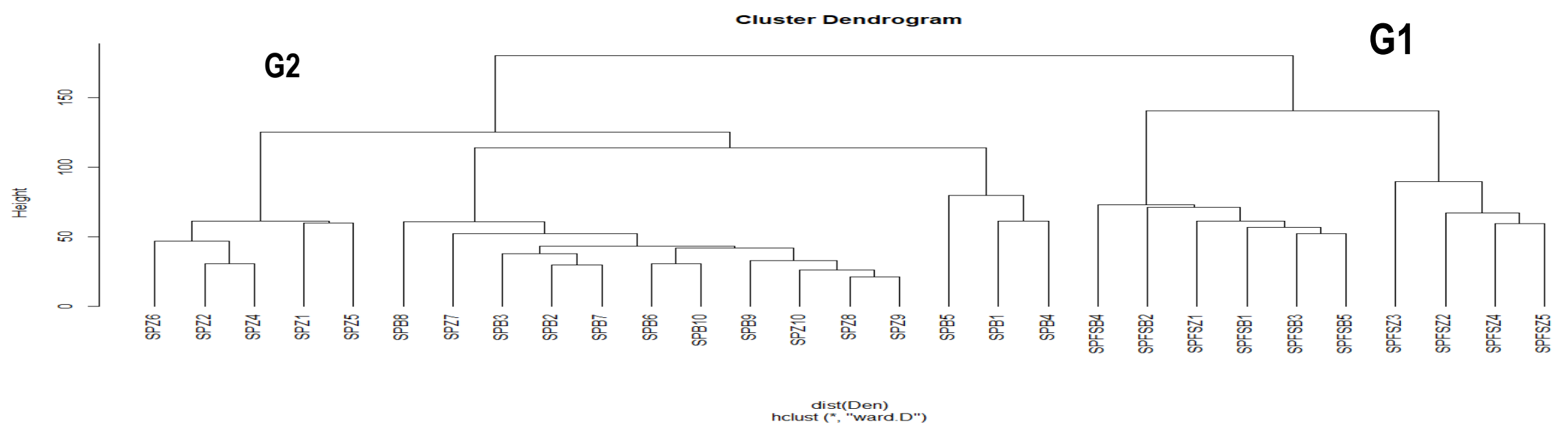

Figure 8 : Dendrogramme des relevés phytosociologiques.

Tableau 1 : Caractéristiques floristiques et diversité spécifique des groupements végétaux.

\begin{tabular}{|c|c|c|c|c|c|c|c|c|c|c|}
\hline \multirow{2}{*}{$\begin{array}{l}\text { Groupements } \\
\text { végétaux }\end{array}$} & \multirow[t]{2}{*}{ Relevés } & \multicolumn{2}{|c|}{ Nombre d'espèces } & \multirow[t]{2}{*}{ Genres } & \multirow[t]{2}{*}{ Familles } & \multicolumn{2}{|c|}{ Paramètres Ecologiques } & \multirow[b]{2}{*}{$\mathbf{E}$} & \multirow{2}{*}{$\begin{array}{c}\text { Familles } \\
\text { dominantes }\end{array}$} & \multirow{2}{*}{$\begin{array}{l}\text { Familles } \\
\text { Dicotylédones }\end{array}$} \\
\hline & & Ligneuses & Herbacées & & & RS & $\mathbf{H}$ & & & \\
\hline $\begin{array}{l}\text { Forêt } \\
\text { Sacrées (G1) }\end{array}$ & 10 & 47 & 83 & 74 & 46 & $130 \pm 0,98$ & $5,92 \pm 0,46$ & $0,97 \pm 0,40$ & $\begin{array}{c}48 \% \\
\text { Leguminoseae } \\
\text { Euphorbiaceae }\end{array}$ & $63 \%$ \\
\hline $\begin{array}{l}\text { Champs/ } \\
\text { Jachères (G2) }\end{array}$ & 20 & 28 & 41 & 42 & 28 & $69 \pm 0,88$ & $3,40 \pm 0,93$ & $0,62 \pm 0,19$ & $\begin{array}{c}22 \% \\
\text { Leguminoseae } \\
\text { Euphorbiaceae }\end{array}$ & $51 \%$ \\
\hline
\end{tabular}


Tableau 2 : Indices de diversité intracommunautaires.

\begin{tabular}{|c|c|c|c|c|c|c|c|c|}
\hline \multirow{4}{*}{$\begin{array}{c}\text { Zones } \\
\text { Guinéo-congolaise }\end{array}$} & \multicolumn{4}{|c|}{ D. arborea } & \multicolumn{4}{|c|}{ N. laevis } \\
\hline & \multicolumn{2}{|c|}{ G1 } & \multicolumn{2}{|c|}{ G2 } & \multicolumn{2}{|c|}{ G1 } & \multicolumn{2}{|c|}{ G2 } \\
\hline & $\mathbf{H}$ & $\mathbf{E}$ & $\mathbf{H}$ & $\mathbf{E}$ & $\mathbf{H}$ & $\mathbf{E}$ & $\mathbf{H}$ & $\mathbf{E}$ \\
\hline & $5,01 \pm 0,87$ & $0,91 \pm 0,04$ & $3,62 \pm 0,17$ & $0,39 \pm 0,09$ & $3,84 \pm 0,12$ & $0,42 \pm 0,02$ & $5,83 \pm 0,36$ & $0,94 \pm 0,07$ \\
\hline Soudano-guinéenne & $5,50 \pm 0,73$ & $0,89 \pm 0,03$ & $3,05 \pm 0,31$ & $0,31 \pm 0,08$ & $3,15 \pm 0,56$ & $0,36 \pm 0,07$ & $5,56 \pm 0,39$ & $0,91 \pm 0,02$ \\
\hline Soudanienne & - & - & - & - & $2,27 \pm 0,33$ & $0,23 \pm 0,16$ & $2,78 \pm 0,43$ & $0,26 \pm 0,18$ \\
\hline
\end{tabular}

(N. laevis : Newbouldia laevis ; D. arborea : Dracaena arborea) $; \mathrm{G}_{1}$ : Forêts sacrées ; $\mathrm{G}_{2}$ : Champs /Jachères ; H : Indice de diversité de Shannon ; $\mathrm{E}$ : Indice d'équitabilité de Pielou.

Tableau 3 : Densité moyenne de tiges à l'hectare chez D. arborea et $N$. laevis.

\begin{tabular}{|c|c|c|c|c|c|c|}
\hline \multirow[b]{2}{*}{$\begin{array}{l}\text { Groupements } \\
\text { végétaux }\end{array}$} & \multicolumn{2}{|c|}{ Guinéo-congolaise } & \multicolumn{2}{|c|}{ Soudano-guinéenne } & \multicolumn{2}{|c|}{ Soudanienne } \\
\hline & $\begin{array}{c}\text { D. arborea } \\
\left(\text { Nbre tiges.ha }^{-1}\right)\end{array}$ & $\begin{array}{c}\text { N. laevis } \\
\left.\text { (Nbre de tiges.ha }^{-1}\right)\end{array}$ & $\begin{array}{c}\text { D. arborea } \\
\text { (Nbre de tiges.ha }^{-1} \text { ) }\end{array}$ & $\begin{array}{c}\text { N. laevis } \\
\text { (Nbre de tiges.ha }^{-1} \text { ) }\end{array}$ & $\begin{array}{c}\text { D. arborea } \\
\left(\text { Nbre de tiges.ha }^{-1}\right)\end{array}$ & $\begin{array}{c}\text { N. laevis } \\
\left.\text { (Nbre de tiges.ha }^{-1}\right)\end{array}$ \\
\hline$G_{l}$ & $64 \pm 08$ & $37 \pm 06$ & $31 \pm 09$ & $11 \pm 02$ & - & $07 \pm 04$ \\
\hline $\mathrm{G}_{2}$ & $28 \pm 11$ & $89 \pm 14$ & $16 \pm 05$ & $56 \pm 10$ & - & $13 \pm 05$ \\
\hline
\end{tabular}



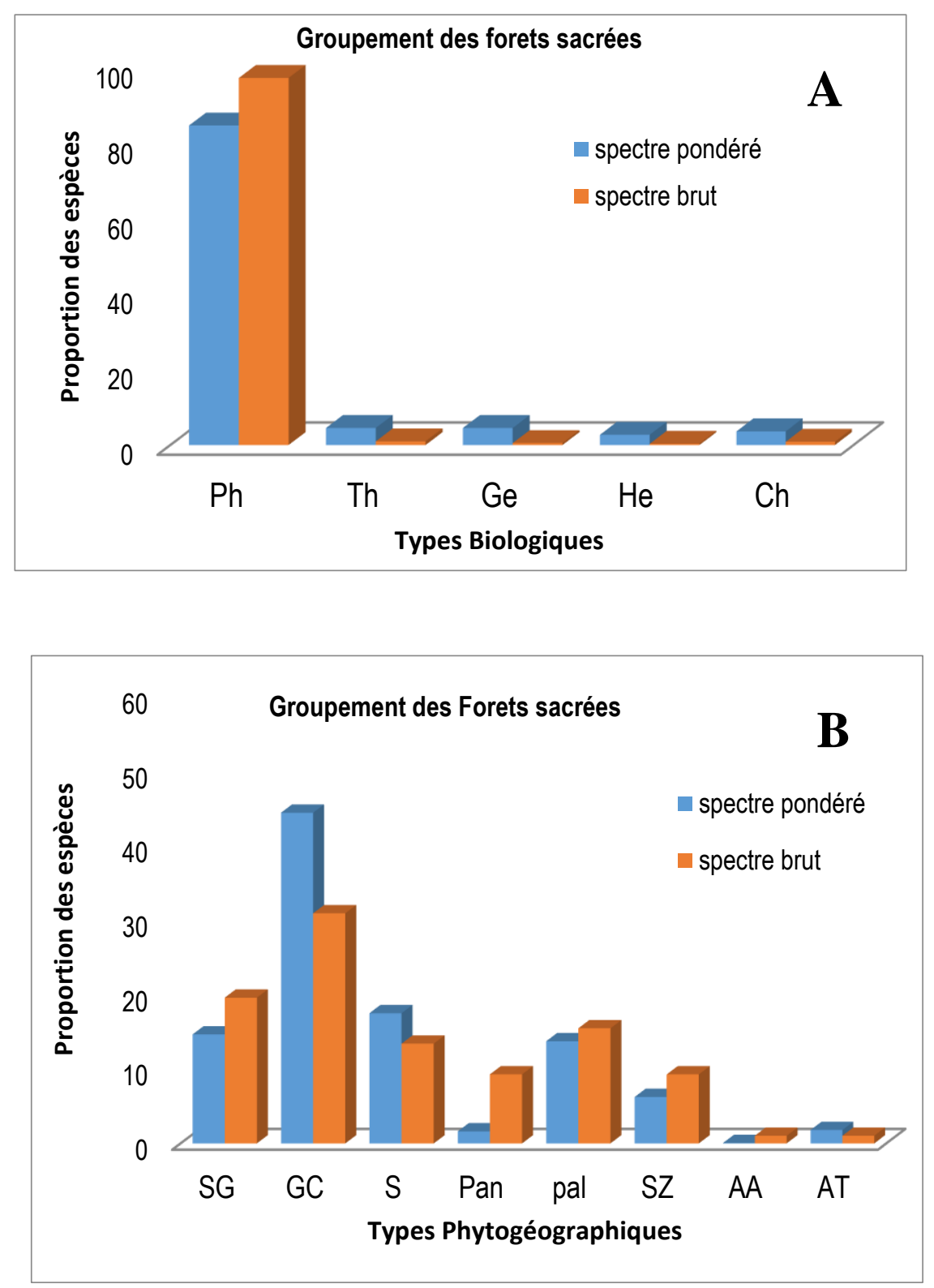

Figure 9: Spectres des types biologiques (9A) et phytogéographique (9B) des groupements de végétaux de forêts sacrées.

Ph: Phanérophytes ; Th: Thérophytes ; Ge: Géophyte ; He: Hémicryptophytes ; Ch: Chaméphytes ; SG : espèces soudanoguinéennes; GC : espèces guinéo-congolaises ;

$\mathrm{S}$ : espèces soudaniennes ; Pan : espèces pantropicales ; Pal : espèces paléotropicales ; SZ : espèces soudano-zambéziennes; AA : espèces afro-américaines ; AT : espèces réparties dans toute l'Afrique tropicale. 

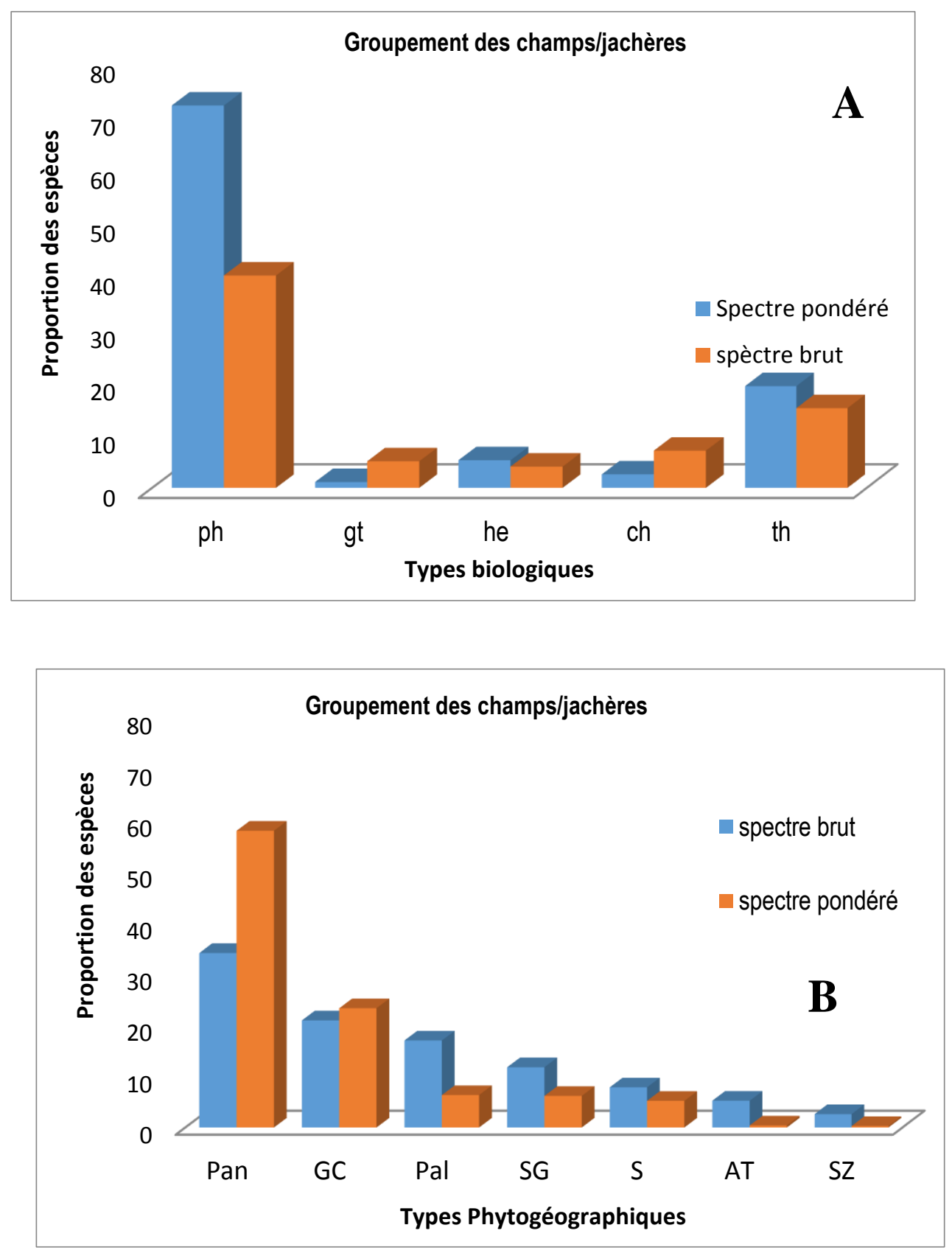

Figure 10: Spectres des types biologiques (10A) et phytogéographique (10B) des groupements végétaux de champs/jachères.

$\mathrm{Ph}$ : Phanérogame ; Th : Thérophyte ; Ge : Géophyte ; He: Hémicryptophytes;

Ch : Chamephyte ; Pan : espèces pantropicales ; GC : espèces guinéo-congolaises ; Pal : espèces paléotropicales ; SG : espèces soudano-guinéennes; $\mathrm{S}$ : espèces soudaniennes; $\mathrm{SZ}$ : espèces soudano-zambéziennes ; AT : espèces réparties dans toute l'Afrique tropicale. 


\section{DISCUSSION}

$\mathrm{La}$ distribution géographique et l'abondance de toute ressource font partie des premières études dans l'évaluation, la potentialité et la disponibilité des espèces (Assongba et al., 2014).

Ainsi, la distribution géographique de $N$. laevis a montré respectivement en zones guinéo-congolaise, soudano-guinéenne et soudanienne des pourcentages des points d'occurrence de $84 \%, 15 \%$ et $1 \%$ et regroupant neuf (09) phytodistricts (Plateau, côtier, vallée de l'Ouémé, Pobè, Zou, Bassila, Borgou sud, chaîne de l'Atacora et Borgou nord). Chez $D$. arborea, les pourcentages des points d'occurrence sont de $83 \%$ en zone guinéenne et $17 \%$ en zone soudano-guinéenne et se répartissent dans six (06) phytodistricts (Côtier, Plateau, Vallée de l'Ouémé, Pobè, Zou et Bassila). De faibles points d'occurrence sont observés en zone soudanienne. La comparaison de la distribution des points d'occurrence sur l'ensemble des phytodistricts donne un large spectre de distribution à $N$. laevis. Ce résultat pourrait s'expliquer par la capacité de $N$. laevis à s'adapter aux différents types de sols des zones bioclimatiques et agrosystèmes des phytodistricts traversés, montrant ainsi que la distribution des espèces végétales n'est pas aléatoire et se fait sous l'influence des facteurs abiotiques (Toko et al., 2014). Les sols de la zone d'étude sont caractérisés par une grande variabilité. $N$. laevis et de $D$. arborea sont identifiés dans des phytodistricts à sols hydromorphes (vallées, cuvettes et plaines alluviales), à vertisols et à sols à caractères vertiques (dépressions médianes), sols à minéraux bruts peu évolués et à affleurements rocheux (plateau, vallée de l'ouémé, littoral, pobè et zou). N. laevis est rencontré sur les sols ferrugineux tropicaux à formations granitogneissiques et sur les schistes des phytodistricts de la chaîne de l'Atacora et du Brogou sud. Les facteurs écologiques influencent la répartition géographique des espèces en agissant sur la texture, l'humidité, la topographie du sol et sur la densité des espèces. Ce résultat corrobore les observations faites sur la distribution géographique de certaines espèces dont Pentadesma butyracea (Avocevou-Ayisso, 2011), Tamarindus indica (Fandohan, 2011), Sclerocarya birrea (Gouwakinnou et al., 2011), Dialium guineensis dans le phytodistrict de Pobè (Assongba et al., 2014), Borassus aethiopum (Gbesso et al., 2014). Cette distribution des espèces pourrait s'expliquer aussi par l'importance culturelle et cultuelle que les populations accordent à certaines espèces dont N. laevis (Dassèkpo et al., 2017 ; Adomou et al., 2018) et D. arborea (Logbo et al., 2019) au cours de leurs déplacements en quête de mieux être. Il ressort de l'analyse de la matrice issue des relevés, les groupements végétaux $\mathrm{G}_{1}$ et $\mathrm{G}_{2}$ qui caractérisent respectivement les forêts sacrées et les champs/jachères. Ce nombre de groupements végétaux est relativement faible par rapport à celui noté dans les études antérieures sur la diversité écologique de certaines formations végétales constituées de forêts, de forêts sacrées, de champs et de jachères (Moumouni et al., 2018 ; Moumouni et al., 2019). Ces différences observées dans le nombre des groupements végétaux pourraient s'expliquer par l'anthropisation à outrance qui caractérise ces groupements végétaux étudiés et par le caractère anthropophile des deux espèces étudiées qui s'y trouvent (Dassèkpo et al., 2017 ; Adomou et al., 2018 ; Logbo et al., 2019). L'étude de la diversité floristique d'une forêt donne une idée sur la diversité spécifique et sur l'ensemble des espèces végétales qui la constituent. Les richesses spécifiques des groupements végétaux ont permis de dénombrer cent quatre-vingt-dix-neuf (199) espèces, réparties en 116 genres et 74 familles. La plus faible richesse spécifique est notée dans le groupement végétal $\mathrm{G}_{2}$ avec 42 genres, 28 familles et 69 espèces. La formation végétale $\mathrm{G} 1$ dispose de la plus grande richesse spécifique avec 74 genres, 46 familles et 130 espèces. Cette variation dans les valeurs des richesses spécifiques pourrait s'expliquer par la différence dans les saisons de collecte des données, du nombre des placettes prospectées (Issoufou, 2011 ; Mamadou et al., 2020), par 
les fortes influences externes que subissent les champs/jachères par rapport aux forêts sacrées. Les perturbations subies par les champs/jachères occasionnées par les feux de brousse, affectent fortement la densité des plantules, la croissance des arbres (Sinsin et al., 2015; Dicko et al., 2016), la strate arborescente et entrainent la prolifération des herbacées aux dépens des arbres, une diminution au cours du temps des espèces originelles et l'apparition de nouvelles espèces constituées pour la plupart d'espèces allochtones. Ces observations corroborent celles obtenues par Folahan et al. (2018) en étudiant l'écologie et la structure des groupements végétaux des écosystèmes de la Lama et de Gbesso et al. (2018) dans la caractérisation de la flore et de la végétation du site archéologique d'Agongointo et de sa périphérie. Les ouvertures créés par ces perturbations au niveau des formations végétales offrent suffisamment de lumière pour le développement des herbacées (Ouattara et al., 2016). La mise en place des exploitations céréalières et pérennes entrâne aussi une réduction de la densité du couvert ligneux et une modification de la composition floristique et édaphique (Toko, 2013). Ces modifications notées, corroborent celles identifiées dans les phytocénoses culturales et post-culturales (Assongba et al., 2014). Des auteurs ont montré que la variation du nombre d'espèces est sous la dépendance des variables stationnaires telles que la géomorphologie, la texture du sol (Mamadou et al., 2020), les unités morphopédologiques (Hakizimana et al., 2012) et qui ont une influence significative dans la distribution des groupements végétaux.

Les résultats montrent en zone guinéocongolaise, des indices de diversité de Shannon et d'équitabilité de Pielou sont respectivement moyen et faible au sein de la communauté végétale $\mathrm{G}_{1}(\mathrm{H}=3,84$ bits; $\mathrm{E}=0,42)$. Par contre, dans le groupement végétal $\mathrm{G}_{2}(\mathrm{H}=5,83$ bits; $\mathrm{E}=0,94)$, ces deux indices sont élevés et dont les espèces dominantes sont Newbouldia laevis (P.Beauv.) Seem. ex Bureau et Elaeis guineensis Jacq. Les résultats observés en zone guinéo-congolaise pourraient s'expliquer par la dominance d'une espèce au détriment des autres espèces dans le groupement végétal $\mathrm{G}_{1}$. Cette répartition se fait de façon irrégulière et non homogène. Dans le groupement végétal $\mathrm{G}_{2}$, la répartition des espèces est homogène et se fait de façon équitable. Par contre, chez $D$. arborea, les indices de diversité de ShannonWiener et d'équitabilité de Pielou sont respectivement moyen et faible dans la communauté végétale $\mathrm{G}_{2}(\mathrm{H}=3,62$ bits et $\mathrm{E}=$ $0,39)$ et élevés dans celle de $\mathrm{G}_{1}$ ( $\mathrm{H}=5,01$ bits; E=0,91) avec Albizia zygia et Triplochiton scleroxylon comme étant les espèces caractéristiques. Par contre, en zone soudanoguinéenne, les indices de diversité de ShannonWiener et d'équitabilité de Pielou sont élevés dans la communauté végétale $\mathrm{G}_{1}(\mathrm{H}=5,50$ bits; $\mathrm{E}=0,89)$ et faibles dans celle de $\mathrm{G}_{2}(\mathrm{H}=3,05$ bits; $\mathrm{E}=0,31$ ) chez $D$. arborea. Ces indices de diversité de Shannon-Wiener et d'équitabilité de Pielou sont faibles $(\mathrm{H}=3,15$ bits; $\mathrm{E}=0,62)$ dans le groupement végétal $\mathrm{G}_{1}$ et élevés $(\mathrm{H}=5,56$ bits; $\mathrm{E}=0,91)$ dans celui de $\mathrm{G}_{2}$. Ces résultats pourraient s'expliquer par la faible diversification de la flore rencontrée dans les champs/jachère par rapport aux forêts sacrées qui contiennent un nombre important d'espèces ayant des recouvrements de même importance. Ces résultats corroborent les observations de Savadogo et al. (2018) qui ont noté dans une matrice de 142 relevés, 350 espèces dans les forêts sacrées et les savanes environnantes dans le secteur sud-soudanien du Burkina Faso ayant des recouvrements de même importance et de Gbesso et al. (2018) qui ont montré que les fortes valeurs de l'indice de Shannon traduisent des recouvrements de même importance chez un nombre important d'espèces. Les faibles valeurs du même indice prouvent, que quelques espèces seulement disposent de forts recouvrements et ceci pourrait être dû à des différences des perturbations climatiques et zoo-anthropiques entre les zones bioclimatiques (Mamadou et al., 2020). La bonne répartition des espèces, serait la conséquence d'une longue évolution des communautés dans des milieux stables (Kidjo et al., 2011). 
La variation de la densité des tiges à l'hectare, montre chez $N$. laevis que les plus grandes densités des tiges à l'hectare sont de 89 tiges.ha ${ }^{-1}$ en zone guinéo-congolaise et de 56 tiges.ha ${ }^{-1}$ en zone soudano-guinéenne dans les groupements végétaux $\mathrm{G}_{2} \quad$ contre respectivement 30 tiges.ha $^{-1}$ et 11 tiges.ha $^{-1}$ dans les zones guinéo-congolaise et soudanoguinéenne pour les groupements végétaux $\mathrm{G}_{1}$. Il en résulte que $N$. laevis développe plus de tiges en milieu humide et anthropisé comme les champs/jachères qu'en milieux humide et naturel comme les forêts sacrées. Par contre, les densités des tiges à l'hectare chez $D$. arborea sont de 28 tiges.ha $^{-1}$ en zone guinéocongolaise et de 16 tige.ha $^{-1}$ en zone soudanoguinéenne dans le groupement végétal $\mathrm{G}_{2}$, contre 64 tiges.ha $^{-1}$ et 31 tiges.ha $^{-1}$ respectivement en zones guinéo-congolaise et soudano-guinéenne dans le groupement végétal $\mathrm{G}_{1}$. Dans les zones guinéo-congolaise et soudano-guinéenne, les densités moyennes de tiges sont réduites de moitié. Ce résultat pourrait s'expliquer par l'incapacité des espèces à compétir pour certaines ressources disponibles dont la lumière et l'eau. En milieu humide anthropisé, les espaces sont plus ouverts qu'en milieux humide et naturel dont les grands arbres constituent souvent un facteur limitant pour l'acquisition de certaines ressources. Les espèces sont utilisées comme des plantes de services dans la définition des limites, dans la délimitation des parcelles $(N$. laevis) et dans les forêts sacrées pour matérialiser les lieux de cultes ou abritant une divinité. La réduction du nombre d'individus des deux espèces est observée en partant de la zone guinéo-congolaise à la zone soudanienne. Dans la zone soudanienne, quelques individus de $N$. laevis sont notés dans les jardins de cases et habitations et bénéficient de l'eau provenant des ménages, traduisant ainsi une forme d'adaptation de l'espèce aux conditions climatiques de cette zone.

Les résultats ont également montré les distributions des types biologique et phytogéographique des groupements végétaux hébergeant $N$. laevis et $D$. arborea. Les spectres bruts et pondérés des groupements végétaux $G_{1}$ et $G_{2}$ révèlent les phanérophytes comme étant les formes de vie dominantes. Suivent les thérophytes, les Géophytes, les chaméphytes et les hémicryptophytes avec des différences significatives entre les fréquences et abondances et les recouvrements des types biologiques. $\mathrm{Ce}$ recouvrement des phanérophytes et des thérophytes observé traduit les états de dégradation des groupements végétaux. Ces états pourraient être liés aux feux de végétation, aux différentes formes d'activités anthropiques, aux conditions climatiques et à la pression pastorale. L'abondance et la dominance des phanérophytes montrent la forte représentativité des ligneux dans les deux formations végétales et ceci pourrait constituer selon Moumouni et al. (2019) un espoir pour la reconstitution naturelle des formations végétales dégradées. Des résultats similaires attribuent la prolifération des thérophytes aux conditions biotiques (Daget et al., 2010), à la minceur des sols de la zone d'étude (Moumouni et al., 2018), par les conditions pédologiques des substratums des sols des zones agroclimatiques suite aux facteurs anthropiques entrainant ainsi la dégradation et la perte de la fertilité des sols (Djego et al., 2006) et le développement des espèces à large spectre de distribution, la baisse du nombre d'espèces de chaméphytes et de thérophytes et enfin leur adaptation aux fortes perturbations (Vroh, 2013).

Au Bénin, des auteurs ont montré que la présence des phanérophytes et thérophytes constitue le signe d'une évidence de perturbation des formations naturelles en zone guinéenne (Gbesso et al., 2013), en zone soudano-guinéenne (Aitonou et al., 2016) et en zone soudanienne (Arouna et al., 2016).

Ces résultats sont contraires à ceux de Toko et al. (2013) qui ont montré en conditions stationnelles favorables, l'installation de plusieurs espèces continentales et une faible colonisation des espèces endogènes. Ces résultats semblent aussi se justifier car les champs des cultures sont à domination des thérophytes. 
Les types phytogéographiques traduisent la fidélité des espèces à leur région de confinement (Imorou, 2008 ; Habou et al., 2020) et permettent de juger de la spécificité de la flore. Ainsi, les spectres bruts et pondérés des types phytogéographiques des groupements végétaux $G_{1}$ montrent une forte dominance des espèces guinéo-congolaises, suivies des espèces soudano-guinéennes, paléotropicales, soudaniennes, pantropicales, soudano-zambéziennes, afro-américaines et afrotropicales avec des différences significatives entre les points de fréquence et d'abondance d'une part et entre les recouvrements des types phytogéographiques d'autre part. Par contre, les spectres bruts et pondérés des types phytogéographiques des groupements végétaux $\mathrm{G}_{2}$ montrent que les espèces pantropicales constituent les espèces dominantes, viennent ensuite les espèces guinéo-congolaises, paléotropicales, soudanoguinéennes, soudaniennes, afrotropicales et soudano-zambéziennes avec des différences significatives entre les fréquences et abondances d'une part et entre les recouvrements des types phytogéographiques d'autres part. Les types phytogéographiques obtenus ont montré une bonne représentation des espèces à large distribution telles que les espèces pantropicales, soudaniennes, soudano guinéennes et soudano zambéziennes qui sont des espèces capables de coloniser les milieux dégradés. La dominance des espèces guinéocongolaises dans la zone d'étude en terme de fréquence et d'abondance, pourrait s'expliquer par le fait que ces espèces sont dans leur zone de prédilection. Par contre, la présence des espèces soudano-guinéenne et soudanienne en zone guinéo-congolaise pourrait s'expliquer par l'anthropisation des forêts sacrées à des fins culturelle et cultuelle. Dans les champs et jachères, la forte dominance des espèces pantropicales sur les autres espèces suivies des espèces guinéo-congolaises et Soudanoguinéennes pourrait s'expliquer par le fait qu'il s'agit des zones guinéo-congolaise et soudanoguinéenne d'une part et d'autre part par le fait que les cultures de champs sont d'origines géographiques diverses et donc adaptées aux besoins de l'homme par la création des variétés. Il s'en suit alors une perte de spécificité des formations végétales étudiées. C'est le cas de l'absence de $D$. arborea noté dans les groupements végétaux $G_{1}$ et $G_{2}$ en zone soudanienne qui est loin de traduire une limite de sa répartition en fonction des paramètres climatiques. Ainsi, Neuenschwander et al. (2011) ont montré que l'eau, le relief, la topographie, la latitude et la longitude constituent des facteurs qui expliquent au mieux la répartition spatiale des espèces. A ces paramètres climatiques, s'ajoutent aussi la variabilité des besoins et les usages des espèces d'une zone à une autre. La distribution de $N$. laevis et de $D$. arborea pourrait être expliquée par le climat, la nature des sols et les actions anthropiques (activités culturales, besoins des populations).

\section{Conclusion}

Cette étude est une contribution à la connaissance de l'écologie, des habitats et des patrons de distribution de Dracaena arborea et de Newbouldia laevis, indispensable à leur conservation dans l'ensemble trois zones bioclimatiques. $\mathrm{La}$ caractérisation phytosociologique a permis d'identifier suivant les traits spécifiques induits par le gradient d'anthropisation, le groupement des forêts sacrées $(\mathrm{G} 1)$ où les espèces expriment le plein de leur potentiel dendrométrique de celui des champs et jachères (G2). Le climat, les types de sols et les actions anthropiques sont les véritables patrons de distribution des espèces. Les espèces s'établissent dans les zones guinéo-congolaise et soudano-guinéenne En zone soudanienne, la rudité du climat ne permet pas un bon établissement des espèces. Toutefois, des individus de Newbouldia laevis sont identifiés en zone soudanienne uniquement autour des habitations servant ainsi pour les besoins de l'homme. En perspective, l'étude des variations des paramètres agromorphologiques des deux espèces suivant les zones bioclimatiques ethnobotanique est envisagée pour montrer les valeurs d'usages et l'importance des deux espèces pour les populations. 


\section{CONFLIT D'INTERETS}

Les auteurs de ce manuscrit déclarent qu'il n'y a aucun conflit d'intérêts.

\section{CONTRIBUTIONS DES AUTEURS}

JL a élaboré le protocole de recherche, a collecté les données sur le terrain, et a rédigé le manuscrit. PY s'est occupé de la détermination des noms des espèces à l'Herbier de l'Université d'Abomey-Calavi. BT a donné des conseils méthodologiques, a révisé le manuscrit et a procédé à la correction du document. L'auteur AA a supervisé les activités de cette recherche et a révisé la version finale du manuscrit. La version finale du manuscrit est approuvée par tous les auteurs.

\section{REFERENCES}

Adjanohoun E, Adjakidjè V, Ahyi MRA, Aké Assi L, Akoegninou A, d'Almeida J, Apovo F, Boukef K, Chadare F, Cusset G, Dramane K, Eyme J, Gassita JN, Gbaguidi N, Goudoté E, Guinko S, Houngnon P, Issa L, Keita A, Kiniffo HV, Koné BD, Musampa NA, Saadou N, Sodogandji T, de Souza S, Tchabi A, Zinsou DC, Zohoun T. 1989. Contribution aux Etudes Ethnobotaniques et Floristiques en République Populaire du Bénin, (2ème édition). ACCT : Paris.

Adomou CA, Dassou GH, Yédomonhan H, Favi GA, Ouachinou JMA, Aboudja MJM, Houenon GAH. 2018. Analyse des connaissances traditionnelles et des déterminants relatifs à l'utilisation de Newbouldia laevis (PBeauv) Seemann ex Bureau (Bignoniaceae) au Sud-Bénin. Afrique SCIENCE, 14(1): 194-205. http://wwwafriquescienceinfo

Adomou AC, Sinsin B, Van der Maesen LJG. 2006. Phytosociological and chorological approaches to phytogeography: a meso-scale study in Benin. Systematics and Geography of Plants, 76: 155-178.

Aïtondji AL, Toyi MSS, Kassa B, Sinsin B. 2016. Caractéristiques Floristiques,
Phytosociologiques et Écologiques de la végétation des carrières en République du Bénin Science de la vie, de la terre et agronomie. Rev. Cames, $\mathbf{0 3}(02)$.

Aké-Assi E, Abdou Yao CY, Ipou Ipou J, Neuba DF, Aké-Assi L, Traoré D. 2010. Représentations des plantes ornementales pour les populations d'Abidjan et San Pedro, en Côte d'Ivoire. In Systématique et conservation des plantes africaines, van der Burgt X, van der Maesen J, Onana J-M (eds). Royal Botanic Gardens : Kew ; 289-296.

Akoègninou $\mathrm{A}$, van der Burg $\mathrm{WJ}$, van der Maesen LJG, Adjakidjè V, Esseou JP, Sinsin B, Yédomonhan H. 2006. Flore Analytique du Bénin. Backhuys Publishers: Cotonou et Wageningen.

Allabi AC, Busiac K, Ekanmiana V, Bakiono F. 2011. The use of medicinal plants in self-care in the Agonlin region of Benin. J. Ethnoph., 133: 234-243.

Assogbadjo AE. 2006. Importance socioéconomique et étude de la variabilité écologique, morphologique, génétique et biochimique du baobab (Adansonia digitata L.) au Bénin. Thèse de doctorat, Faculteit Bio-Ingenieur-swetenschappen, Universiteit Gent, Gent, 213p.

Assongba YF, Déléké-koko I, Yoka J, Djègo JG, Sinsin B. 2014. Caractérisation des habitats de Dialium guineense (Willd) en République du Bénin. J. Appl./ Biosci., 83: 7520-7534. http://dxdoiorg/104314/jabv83i11.

Avocevou Ayisso C, AvohouTH, Oumorou M, Dossou G, Sinsin B. 2011. Ethnobotany of Pentadesma butyracea Sabine in Benin: A quantitative approach. Ethnobot. J., 9: 1547-3465. www.ethnobotanyjournal.org vol3/i1547-3465-03-025.pdf.

Awono A, N'doye O. 2003. Les vertus des PFNL dans la zone forestière humide du Cameroun et l'impact de leur exploitation sur l'environnement. WFC - XII- 0710 A2.

Chibembe AS, Birhashirwa NR, Kamwanga F, Mangambu M. 2015. Exploitation de 
Bambous (Sinarundinaria alpina (K Schum) CS Chao et Renvoize), cause des conflits entre le Parc National de KahuziBiega et la population environnante : stratégie de conservation et de résolution de Conflit. Int. J. Env. St., 72(2): 265-287. DOI: $10.1080 / 00207233.2014 .961270$

Daget P, Possonet J. 2010. Prairies et Pâturages: Méthodes d'Etude de Terrain et Interprétations. CIRAD.

Dassekpo SI, Achigan-dako EG, Honfo HS, Tovissodé F, Tenté B, Houssou CS, Ahanchédé A. 2017. Approches Socioculturelle et Culturelle des Usages de Newbouldia laevis et plantes associées dans les traditions du Bénin. Rev. Ivoir. Sci. Technol., 30(2017): 291-304. http://www.revist.ci.

Dicko A, Biaou SSH, Natta AH, Gado CAS, Kouagou M. 2016. Influence des pressions anthropiques sur la structure des populations de Pentadesma butyracea au Bénin. VertigO - la revue électronique en sciences de l'environnement, 16(3). DOI : $10.4000 /$ vertigo. 18195

Djègo J, Sinsin B. 2006. Impact des espèces exotiques plantées sur la diversité des phytocénoses de leur sous-bois. Système Géographique, 76: 191-209.

https://www.jstor.org/stable/20649711.

Fandohan B, Assogbadjo AE, Kakaï Glèlè R, Sinsin B. 2011. Geographical distribution, tree density and fruit production of Tamarindus indica L. (Fabaceae) across three ecological regions in Bénin. Fruits, 66(2): 65-78. DOI: https://doi.org/10.1051/fruits/2010043.

Fatunla OA, Ogundare AO, Achimugu II, Akindele PO. 2016. Antibacterial Effect of Newbouldia laevis Leaf Extract on Vancomycin and Methicillin Resistant Bacterial Isolates from Federal Medical Center, Owo. J. Adv. Microbiol., 1(3): 111. wwwsciencedomain.org.

Farnsworth EJ, Meyerson LA. 1999. Species composition and inter-annual dynamics of a freshwater tidal plant community following removal of the invasive grass,
Phragmites australis. Biol. Inv., 1: 115127.

DOI: https://doi.org/10.1023/A:101006860763 0.

Folahan SON, Dissou EF, Akouehou GS, Tenté BAH, Boko M. 2018. Ecologie et structure des groupements végétaux des écosystèmes de la Lama au Sud-Bénin. Int. J. Biol. Chem. Sci., 12(1): 322-340. DOI:

https://dx.doi.org/10.4314/ijbcs.v12i1.26

Gbesso F, Logbo J, Lougbegnon OT, Tenté B. 2018. Caractérisation de la flore et de la végétation du site archéologique d'Agongointo et de sa périphérie (Commune de Bohicon, Bénin). Revue Internationale des Sciences Appliquées, 1(02): 39-48.

Gbesso F, Yédomonhan H, Tenté B, Akoegninou A. 2014. Distribution géographique des populations de rôniers (Borassus aethiopum Mart, Arecaceae) et caractérisation phytoécologique de leurs habitats dans la zone soudano-guinéenne du Benin. J. Appl. Biosci., 74: 60996111. DOI : http://dx.doi.org/10.4314/jab.v74i1.14.

Habou MKA, Rabiou H, Karim S, Maazou R, Matchi II, Mahamane A. 2020. Caractéristiques floristique et écologique des formations végétales de Gouré (Sudest du Niger). Rev. Mar. Sci. Agron. Vét., 8(2): 186-195.

Hakizimana P, Bangirinama F, Masharabu T, Habonimana B, De Cannière C, Bogaert J. 2012. Caractérisation de la végétation de la forêt dense de Kigwena et de la forêt claire de Rumonge au Burundi. Bois et Forêts des Tropiques, 312(2): 4352. httsp://bft.cirad.fr/cd/BFT_312_4352.pdf

Ilodibia CV, Ugwu RU, Okeke CU, Akachukwu EE, Ezeabara CA. 2015. Phytochemical evaluation of various parts of Dracaena arborea Link and Dracaena mannii Bak. African J. Plant Sci., 9(7): 287-292. DOI: 105897/AJPS20141258.

Mamadou IM, Habou MKA, Rabiou H, Mahamane A. 2020. Phytodiversité et productivité herbacée du futur site de pré- 
lâché d'autruche d'Afrique du Nord (Struthio camelus camelus L.) dans le Koutous, Niger. Int. J. Biol. Chem. Sci., 14(2): 402-416. DOI: https://dx.doi.org/10.4314/ijbcs.v14i2.8

Issoufou IA. 2011. Etude de faisabilité pour la création d'un site de pré-lâché ou lâcher de l'autruche à cou rouge (Struthio camelus camelus) dans la zone de Kellé à Zinder. Mémoire de Master, Université d'Abomey Calavi, Bénin, 49p.

Juhe-Beaulaton D. 2009. Arbres mémoires, forêts sacrées et jardins des plantes de Porto Novo (Bénin) : un patrimoine naturel urbain à considérer. Autrepart revue de sciences sociales au Sud, 51: 7598.

Kaou KAK, Manzo OL, Karim IDG, Rabiou S, Habou RP. 2017. Diversité floristique et structure de la végétation dans la zone dunaire du sud-est du Niger: Cas de Mainé soroa. J. Appl. Biosci., 120: 1205312066.

DOI : https://dx.doi.org/10.4314/jab.v120i1.8.

Kidjo FC, Lougbegnon OT, Djossa AB, Oumorou M, Codjia JTC, Sinsin B. 2011. Caractérisation phyto-écologique de l'habitat du Tragelaphus spekei gratus (Sclater, 1864), Sitatunga, dans la partie méridionale du Bénin. Int. J. Biol. Chem. Sci., 5(4): 1603-1618. DOI: http://dx.doi.org/104314/ijbcsv5i424.

Kokou K, Couteron P, Martin A, Caballe G. 2002. Taxomonic diversity of lianas and wines in forests fragments of southern Togo. Revue d'Ecologie (Terre Vie), 57: 3-17. http://hdl.handle.net/2042/55476.

Lawin IF, Lalèyè OAF, Agbani OP. 2016. Vulnérabilité et stratégies endogènes de conservation des plantes utilisées dans le traitement du diabète dans les communes de Glazoué et Savè au Centre-Bénin. Int . J. Biol. Chem. Sci., 10(3):1069-1085. DOI:

http://dxdoiorg/104314/ijbcsv10i314

Logbo J, Yédomonhan P, Tenté B, Akoegninou A. 2019. Usages et importances culturelles de Dracaena arborea (Willd) Link chez les peuples Kwa au Bénin.
Afrique SCIENCE, 15(2): 117-132. http://wwwafriquesciencenet.

Mangambu M, Robbrecht E, Ntahobavuka H, van Diggelen R. 2015. Analyse phytogéographique des Ptéridophytes d'Afrique centrale: cas des étages des montagnes du Parc National de KahuziBiega (République Démocratique du Congo). European Scientific Journal, 10 (8): 84-106.

Masumbuko C, Habiyaremye F, Lejoly J. 2013. Impact of Sericostachys scandens on forest regeneration in the KahuziBiega National Parc. In African Plant diversity, Beau N, Dessein S, Robbrecht E (eds). Scipta Botanique: Belgica; 130137.

Moumouni YI, Arouna O, Zakari S. 2018. Diversité Floristique et Structure des formations végétales dans le district phytogéographique du Borgou-Nord au Bénin (Secteur de l'Arrondissement de Bagou). Notes Scientifiques (Homme et Société), Faculté des Sciences de l'Homme et de la Société, Université de Lomé, p. 63-80.

Moumouni YI, Toko II, Moussa AD. 2019. Caractérisation de la Dégradation à Travers la Diversité Floristique et la Structure de la Végétation dans le Bassin Moyen de la Sota au Nord-Bénin. International Journal of Progressive Sciences and Technologies, 18(1): 53-70. http://ijpsat.ijsht-journals.org.

Neuenschwander P, Sinsin B, Goergen G. 2011. Protection de la Nature en Afrique de l'Ouest : Une Liste Rouge pour le Bénin Nature Conservation in West Africa : Red List for Benin (eds). International Institue of Tropical Agriculture : Ibadan, Nigeria.

Ouattara D, Kouamé D, Tiebré MS, Cissé A, N'guessan KE. 2016. Diversité floristique et usages des plantes dans la zone soudanienne du Nord-ouest de la Côte d'Ivoire. Journal of Animal \& Plant Sciences, $\quad 31(1): \quad 4815-4830$. http://wwwmelewaorg/JAPS. 
Raunkiaer C. 1934. The Life Forms of Plants and Statistical Plant Geography. Clarendon Press: Oxford.

Salhi S, Fadli M, Zidane L, Douira A. 2010. Etudes floristique et ethnobotanique des plantes médicinales de la ville de Kénitra (Maroc). J. Laz., 31: 133-146. DOI:10.5209/rev_LAZA. 2010.v31.9.

Savadogo S, Sambaré O, Thiombiano A. 2018. Écologie et diversité des bois sacrés et des savanes environnantes du secteur sudsoudanien du Burkina Faso (Afrique de l'Ouest). Journal of Animal \& Plant Sciences, $\quad 35(3)$ : $\quad 5715-5733$. http://wwwmelewaorg/JAPS.

Sinsin F, Glèlè Kakaï R, Orthmann B, Sinsin B. 2015. Tree-ring: a suitable implement for spatial and temporal fire distribution analysis in savanna woodland and dry forest. Journal of Forestry research, 26(2) : 435-446. DOI: 10.1007/s11676015-0047-7.

Toko II. 2013. Effets des facteurs abiotiques sur la répartition spatiale des groupements végétaux dans la zone de transition soudano-guinéenne du Bénin,
Int. J. Biol. Chem. Sci., 7(6): 2178-2192. DOI : http://dxdoiorg/104314/ijbcsv7i6

Toko MI, Toko II, Gbegbo MC, Sinsin B. 2013. Structure et composition floristiques des forêts denses sèches de la région des Monts Kouffé au Bénin. $J$. Appl. Biosci., 64: 4787-4796. DOI : http://dx.doi.org/10.4314/jab.v64i1.8846 7.

Vroh BTA. 2013. Évaluation de la dynamique de la végétation dans les zones agricoles d'Azaguié (Sud-est Côte d'Ivoire). Thèse de Doctorat unique, UFR Biosciences, Université Félix Houphouët-Boigny, Abidjan, Côte d'Ivoire, $163 \mathrm{p}$.

Wédjangnon AA, Houètchégnon T, Ouinsavi C. 2016. Caractéristiques ethnobotaniques et importance socioculturelle de Mansonia altissima A Chev au Bénin, Afrique de l'Ouest. Journal of Animal \& Plant Sciences, 29(3): 46784690. http://www.m.elewa.org/JAPS.

White F. 1986. La végétation de l'Afrique: mémoire accompagnant la carte de végétation de l'Afrique. ORSTOM: Paris. 